\title{
A new model for wave-induced instantaneous liquefaction in a non-cohesive seabed with dynamic permeability
}

\author{
Mo-Zhen Zhou ${ }^{\mathrm{a}}$, Dong-Sheng Jeng ${ }^{\mathrm{b}}$, Wen-Gang Qi ${ }^{\mathrm{c}, \mathrm{d}, *}$ \\ ${ }^{a}$ Key Laboratory for Urban Underground Engineering of the Ministry of Education, Beijing Jiaotong University, Beijing, 100044, China \\ ${ }^{\mathrm{b}}$ School of Engineering \& Built Environment, Griffith University Gold Coast Campus, Queensland, 4222, Australia \\ ${ }^{c}$ Key Laboratory for Mechanics in Fluid Solid Coupling Systems, Institute of Mechanics, Chinese Academy of Sciences, Beijing, 100190, China \\ ${ }^{\mathrm{d}}$ School of Engineering Science, University of Chinese Academy of Sciences, Beijing, 100049, China
}

\section{A R T I C L E I N F O}

\section{Keywords:}

Dynamic permeability

Excess pore pressure

Soil liquefaction

Wave-seabed interaction

\begin{abstract}
A B S T R A C T
Wave-induced instantaneous liquefaction is crucial to evaluating the stability of offshore foundations. For the instantaneous liquefaction, most previous studies treated the seabed as an invariant poro-elastic medium and hence led to nonphysical tensile stress in the non-cohesive liquefied zone. In this study, a penalty-like dynamic permeability model is proposed, based on the experimental evidences of permeability increase during soil liquefaction. The nonlinearity induced by the dynamic permeability is solved by an iterative procedure, based on the Newton-Raphson method. The numerical procedure is implemented in an in-house code and compared with analytical solutions under the constant permeability assumption. Numerical observations validate that the proposed dynamic permeability model can alleviate or even eliminate the nonphysical tensile stress in the instantaneously-liquefied zone. In contrast to the constant permeability model, the new model with a dynamic permeability obtains a better agreement with existing cylinder tests under one-dimensional wave loading conditions. For the numerical examples presented, the liquefaction depths predicted by constant permeability can be up to twice of those by the new dynamic permeability model. It is concluded that the conventional model with constant permeability overestimates the liquefaction potential.
\end{abstract}

\section{Introduction}

Waves propagating over the seabed will induce an excess pore pressure and a consequent decrease of the effective stress in marine sediments. In the wave-dominated coastal locations or the surf zones, seabed liquefaction can even occur under some extreme wave conditions. The wave-induced pore pressure and associated seabed liquefaction have been widely recognized as critical factors in analyzing the stability of foundations for offshore installations. It has been observed from the laboratory experiments and offshore in-situ measurements that the wave-induced pore pressure can be generally divided into two categories: the residual and oscillatory pore pressures (Zen and Yamazaki, 1990; Sumer, 2014; Jeng, 2018). The residual pore pressure, i.e., the accumulated excess pore pressure, is mainly induced by the compression tendency of soil skeleton under cyclic wave loading. The oscillatory or transient pore pressure is mainly characterized by the amplitude damping and phase lag along the depth in an unsaturated seabed (but with high saturation degree) (Sakai et al., 1992). For details about the residual pore pressure and associated progressive liquefaction, readers can refer to Sassa and Sekiguchi (2001), Sumer et al. (2006), Sumer (2014) and Jeng (2018) or other literatures. This study mainly focuses on the oscillatory pore pressure and associated instantaneous liquefaction.

On the basis of Biot's poro-elastic consolidation theory (Biot, 1941), numerous analytical solutions have been obtained for wave-induced oscillatory pore-pressure response since the 1970s. Among these, Yamamoto et al. (1978) proposed a concise closed-form solution for an isotropic, poro-elastic and infinite seabed. A similar work with anisotropy permeability was reported by Madsen (1978). The effects of finite thickness and three-dimensional short-crested wave were further considered by Hsu and Jeng (1994). Another different approximation based on the theory of mixture, i.e., the boundary layer approximation, was proposed by Mei and Foda (1981), which was found to perform better in fine sand than coarse sand (Hsu and Jeng, 1994). These

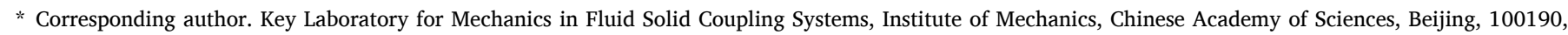
China.

E-mail address: qiwengang@imech.ac.cn (W.-G. Qi).
} 
analytical solutions provided a solid basis to understand the wave-induced oscillatory soil response, however, they have been limited to the cases without any structure. For the cases with a structure, the numerical modeling becomes a better option (Qi et al., 2019). This issue has attracted an increased interest in recent years for various applications, including offshore wind turbine foundations (Sui et al., 2016, 2017; Lin et al., 2017; Li et al., 2018, 2020), pipelines (Li et al., 2019; Qi et al., 2020), breakwaters (Jeng et al., 2001; Zhang et al., 2018; Celli et al., 2019) and immersed tunnel (Chen et al., 2019; Han et al., 2019).

A major limitation of the above studies is that negative effective stresses (i.e., tensile stresses) can be resulted in the instantaneouslyliquefied soil. However, tensile behavior is nonphysical in a noncohesive seabed (Qi and Gao, 2018) and can further significantly pollute the overall pore pressure distribution, as presented in Section 4. These nonphysical tensile stresses during the instantaneous liquefaction may originate from the invariant poro-elastic assumption adopted by existing models (Yamamoto et al., 1978; Sui et al., 2016). These models commonly omitted the variation of physical and mechanical parameters in seabed during liquefaction, such as the soil modulus, permeability, etc. Experimental observations have ever indicated that soil modulus decreases with effective stress by power law at very low effective stress (Haigh et al., 2012; Adamidis and Madabhushi, 2016), implying a nearly zero-value soil modulus while liquefaction occurs. There are experimental evidences for dramatic increase of soil permeability when effective stresses reduce to zero during soil liquefaction (Arulanandan and Sybico, 1992; Ha et al., 2003; Haigh et al., 2012; Wang et al., 2013; Shahir et al., 2014; Ueng et al., 2017). Therefore, there are generally three ways to alleviate or avoid the aforementioned nonphysical tensile stresses in instantaneously-liquefied seabed: (i) decreasing the solid-phase resistance (e.g., soil modulus), (ii) increasing the fluid-phase flow capacity (e.g., soil permeability), and (iii) a combination of both treatments.

According to the numerical experiences from implicit quasi-static simulations, two issues must be addressed while handling the loss of solid-phase resistance. One is the nonlinearity introduced by the resistance variation. This issue can be treated by standard nonlinear iterative methods such as the classical Newton-Raphson method. The other issue is an ill condition or singularity of the global matrix system resulted from the zero-value soil modulus. This issue, however, requires nontrivial numerical techniques in addition to standard finite element frameworks. The difficulty can be avoided if the method of increasing the fluid-phase flow capacity is adopted. Only the nonlinearity introduced by the flow capacity variation needs to be treated. Therefore, this paper focuses on utilizing the permeability increase and proposes a dynamic permeability model to alleviate the nonphysical tensile stress in the instantaneouslyliquefied zone.

Notably, there existed studies considering the variation of soil permeability and shear modulus for non-homogeneous seabed (Jeng and Lin, 1996, 1999; Jeng and Seymour, 1997; Zhang et al., 2016; Sui et al., 2019). However, these works only considered the variation in spatial domain and hence fell into the scope of linear fluid flow behavior. Wu and Jeng (2019) was the first considered soil permeability being a function of pore-water pressures in the numerical analysis of wave-seabed interactions. This new model introduced a nonlinear flow behavior, which is essentially different from the aforementioned spatial-variable permeability. It was recently extended by Wu et al. (2020) to further take into account dynamic saturation degree for consolidation-induced contaminate transport. However, the dynamic permeability model used in Wu and Jeng (2019) and Wu et al. (2020) was found to cause an apparent decrease of the soil permeability in the liquefied zone, disagreeing with increased permeability during soil liquefaction observed in existing experiments. Therefore, an appropriate approach for wave-seabed interactions with dynamic soil permeability is desired.

This study aims to present a new dynamic permeability model capable of simulating wave-seabed interactions involving liquefaction.
The present work focuses on the case of pure seabed without a structure, which has a fundamental significance for future works considering offshore foundations. This paper is organized as follows. Section 2 briefly introduces the governing equations by using Biot's poro-elastic theory. In Section 3, the previous models for describing the permeability variation are reviewed and then modified to adapt for waveinduced instantaneous liquefaction. The nonlinearity associated with dynamic permeability is treated by an iterative procedure based on the Newton-Raphson method. The numerical implementation is accomplished in our in-house code. Sections 4 provides numerical validations under one-dimensional (1-D) wave loading conditions and exemplary results under two-dimensional (2-D) wave loading conditions to highlight the significant influence of dynamic permeability on the waveinduced instantaneous liquefaction. These results proved that the new model can greatly alleviate the tensile stress in liquefied zones and provide reliable numerical predictions. The nonlinear convergence performance is also investigated. Some conclusions are drawn in Section 5.

\section{Governing equations}

Fig. 1 shows a seabed consolidation problem under wave loading. In this work, the seabed is under the assumption of porous medium. According to the quasi-static consolidation theory by Biot (1941), the governing equations are given as follows:

$\nabla \cdot\left(\boldsymbol{\sigma}^{\prime}-p \mathbf{I}_{2 \times 2}\right)+\mathbf{b}=\mathbf{0}$

$\frac{\partial \varepsilon_{v}}{\partial t}+n \beta \frac{\partial p}{\partial t}-\nabla \cdot\left(\frac{k}{\gamma_{w}} \nabla p\right)=0$

where the first equation represents the equilibrium of the solid-fluid mixture, with the effective stress $\boldsymbol{\sigma}^{\prime}$, wave-induced excessive pore pressure $p$, a second-order unit tensor $\mathbf{I}_{2 \times 2}$ and body force per unit volume $\mathbf{b}$. The second equation establishes the conservation of mass, with the volumetric strain $\varepsilon_{v}$, consolidation time $t$, soil porosity $n$, pore fluid compressibility $\beta$, Darcy's permeability coefficient $k$ and unit weight of pore water $\gamma_{w}$.

For seabed consolidation problems, the pore fluid compressibility $\beta$ is determined by:

$\beta=\frac{1}{K_{\mathrm{w} 0}}+\frac{1-S_{r}}{P_{\mathrm{abs}}}$

where $K_{\mathrm{w} 0}$ is the true bulk modulus of water and taken as $2 \times 10^{9} \mathrm{~Pa}$ (Yamamoto et al., 1978). $S_{r}$ is the degree of saturation. The absolute water pressure $P_{\mathrm{abs}}$ denotes the local static pore pressure in the seabed, which is commonly represented by $P_{\mathrm{abs}}=\gamma_{w} h$, with $h$ the water depth shown in Fig. 1.

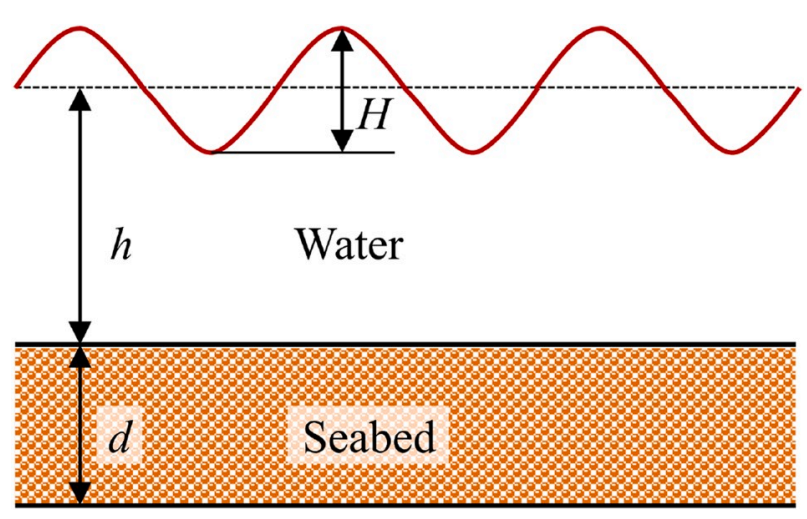

Fig. 1. Schematic of the seabed consolidation problem under wave loading. 


\section{Dynamic permeability models}

The fluid flow in porous media is assumed to obey the generalized Darcy's law, providing the relation between the fluid velocity and pore pressure:

$\mathbf{v}_{w}=-\frac{k}{\gamma_{w}} \nabla\left(-p+p_{z}\right)$

where $p_{z}=\gamma_{w} z$ is the pressure related to position/elevation.

The permeability of the soil is assumed constant or dependent on the spatial location in most previous numerical investigations with respect to wave-induced seabed response. Wu and Jeng (2019) pointed out that the pore pressure $p$ has a significant effect on the soil permeability $k$. The dependence of $k$ on $p$ was considered to analyze the seabed response via a $k-p$ relationship originally proposed by Gardner (1956) for the unsaturated moisture flow equation. An apparent decrease of the soil permeability can be seen in the simulated liquefied zone (Wu and Jeng, 2019), showing a disagreement with increased permeability during soil liquefaction observed in recent studies. The detailed information concerning permeability increase during liquefaction is presented as below.

\subsection{Mechanism of permeability increase during liquefaction}

The theoretical basis of the permeability increase during liquefaction process can be tentatively interpreted by the well-known Kozeny-Carman (KC) equation (Kozeny, 1927; Carman, 1956):

$k=\frac{\gamma_{w}}{\mu} \frac{1}{\kappa_{0} \varsigma S_{0}^{2}} \frac{e^{3}}{1+e}$

with $\mu$ the dynamic viscosity of water, $\kappa_{0}$ the pore shape factor, $S_{0}$ the wetted surface area per unit volume of particles, and $e$ the void ratio of soil. The tortuosity factor of the pore-flow $\varsigma$ is generally defined as the ratio of the actual path length of the fluid particles to the shortest path length in the flow direction (Ghassemi and Pak, 2011). The KC equation provides a link between pore-level media attributes and flow resistance in pore channels. When liquefaction occurs due to seismic or ocean wave loading, with increases of pore pressure and loss of grain contacts, there is negligible volume change of the sand in a short time. Therefore, the values of the void ratio of soil and the unit weight of pore water can be considered approximately constant during liquefaction. However, the values of $\kappa_{0}$ and $\zeta$ will remarkably change during liquefaction, as discussed below.

When soil liquefies, the soil particles lose full contact with each other and are in suspension in the pore fluid. This creates an easier and shorter path for the pore water to readily flow through the liquefied soil without bypassing the grain contacts. The creation of such flow paths reduces the tortuosity $\varsigma$ and the pore shape factor $\kappa_{0}$ (Shahir et al., 2014; Ueng et al., 2017). Hence the soil permeability becomes larger than its initial value. However, the estimation of $\kappa_{0}, S_{0}$ and $\varsigma$ is not straightforward in engineering practice and hence only empirical values are available. A modified KC formula by Liu and Jeng (2019) can avoid the use of these inconvenient parameters. In their study, only particle geometry parameters and the soil porosity are involved. During soil liquefaction, the particle geometry parameters remains constant but the porosity will increase. According to the modified $\mathrm{KC}$ formula, the permeability consequently increases significantly during liquefaction.

The above theoretical analysis can find many experimental evidences in existing literatures. For example, Arulanandan and Sybico (1992) used resistivity measurement in centrifuge tests to characterize the soil structure during and after liquefaction. It was found that the permeability of saturated sand during liquefaction increased to 6-7 times its initial value. Ha et al. (2003) observed in shaking table tests that the sand permeability during liquefaction increased to $1.4-5$ times the initial permeability. Haigh et al. (2012) fluidized the sand specimen with an upward flow (sand boiling) and then lowered the flow rate to measure the permeability. As the effective stress approached zero, the permeability was found to increase 1.1-5 times the original value. Wang et al. (2013) conducted shaking table tests and concluded that the post-liquefaction behavior can be predicted well if the change of permeability after liquefaction, which is about 4 times its initial value, was considered. Ueng et al. (2017) developed a new experimental approach by combining seepage and liquefaction tests together to directly measure the permeability of a saturated sand specimen during and after liquefaction. It was found that the permeability during liquefaction was about 4-5 times the initial value.

Although the above laboratory tests focused on the saturated sand, the qualitative tendency of the permeability increase can be reasonably shared by unsaturated soil. As aforementioned, the KC equation can be used to explain the physical mechanism of permeability increase in the saturated sand. Chapuis and Aubertin (2003) reported that the KC equation is applicable for an unsaturated sand if a reduction factor is introduced. This reduction factor is determined by the saturation degree. Therefore, when liquefaction occurs in an unsaturated seabed, $k_{0}$ and $\square$ will decrease and therefore the permeability increases. It is also worth to conduct laboratory tests specifically designed for wave-induced seabed liquefaction to measure the permeability variation. However, this work only focus on the numerical aspect.

\subsection{Equations for dynamic permeability associated with liquefaction}

Several equations have been proposed for dynamic permeability during liquefaction. In these equations, the dynamic permeability is commonly expressed as a function of excess pore pressure ratio $r_{u}$, being the ratio between the excess pore pressure and initial confining stress. Haigh et al. (2012) presented a power-law expression of the dynamic soil permeability to fit their experimental results of a series of fluidization tests:

$k(p)=k_{0}\left(1-r_{u}\right)^{c}$

where $k_{0}$ is the initial value of the soil permeability and $r_{u}$ is the excess pore pressure ratio. $r_{u}$ is defined as the ratio of excess pore pressure to the initial vertical effective stress, noting that this definition will be modified in Eq. (9) for wave-seabed interactions. The parameter $c$ varies from -0.30 to -0.01 for different potentially liquefiable sands in their experiments. Eq. (5) results in an infinite permeability for the fluidized sand $\left(r_{u}=1.0\right)$. However, only the permeability after the soil liquefaction was measured in their tests. Using the fitting results to extrapolate the soil permeability during liquefaction seems lack of evidence.

Shahir et al. (2014) proposed another expression of the dynamic soil permeability to account for the pore pressure evolution during seismic liquefaction:

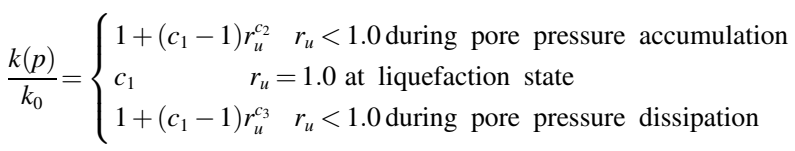

where $c_{1}, c_{2}$ and $c_{3}$ are model parameters. This equation is an improved version based on their previous work (Shahir et al., 2012). Combining benchmark centrifuge tests, they calibrated the constants as: $c_{1}=10$, $c_{2}=2$ and $c_{3}=10$.

Eq. (6) was further modified by Ueng et al. (2017) to fit their experimental data during the pore pressure dissipation phase:

$k(p)=k_{\mathrm{f}}+\left(k_{\mathrm{L}}-k_{\mathrm{f}}\right) r_{u}^{4.8}$

where $k_{\mathrm{f}}$ is the permeability after full dissipation of excess pore pressure, and $k_{\mathrm{L}}$ is the permeability at $r_{u}=1$, i.e., during liquefaction. It was found that $k_{\mathrm{L}}$ is $4-5$ times the initial value $k_{0}$. The permeability variation during pore pressure buildup phase was not mentioned. The difference 
between Eq. (7) and Eq. (6) is that $k_{\mathrm{f}}$ is considered in Eq. (7) rather than the initial permeability $k_{0}$ in Eq. (6).

Based on Eq. (6) and conducting some modifications to adapt for wave-induced liquefaction, the following permeability equation is proposed:

$\frac{k(p)}{k_{0}}= \begin{cases}1 & r_{u} \leq r_{u}^{\mathrm{cr}} \\ 1+\left(c_{1}-1\right)\left(r_{u}-r_{u}^{\mathrm{cr}}\right)^{c_{2}} & r_{u}>r_{u}^{\mathrm{cr}}\end{cases}$

where $c_{1}, c_{2}$ and $r_{u}^{\mathrm{cr}}$ are model parameters. For wave-seabed interaction, the excess pore pressure ratio $r_{u}$ is defined as (see Qi and Gao, 2018):

$r_{u}=\left(p-P_{b}\right) / \sigma_{v 0}^{\prime}$

with $P_{b}$ the dynamic wave pressure at the seabed surface. Assuming that seabed surface is a horizontal plane, the initial vertical effective stress of the seabed $\sigma_{v 0}^{\prime}$ is determined by $\sigma_{v 0}^{\prime}=\gamma^{\prime}|z|$, where $\gamma^{\prime}$ is the buoyant unit weight of the seabed and $z$ is the soil depth.

To provide an intuitive explanation about the modifications of Eq. (8) from Eq. (6), the $k / k_{0}-r_{u}$ curves in both equations are plotted (see Fig. 2). In Fig. 2, the model by Shahir et al. (2014) with $c_{1}=10, c_{2}=2$ and $c_{3}=10$ is labelled as DP1 (Dynamic Permeability). The symbols DP1-a and DP1-d denote the pore pressure accumulation and dissipation phases, respectively. Two different groups of model parameters are considered with respect to the model by Eq. (8): (i) DP2 corresponds to $c_{1}=901, c_{2}=2$ and $r_{u}^{\mathrm{cr}}=0.9$; (ii) DP3 corresponds to $c_{1}=100, c_{2}=1$ and $r_{u}^{\mathrm{cr}}=1$. The value of $c_{1}=901$ in DP2 is chosen in such a way that DP2 and DP1 have an equal value of permeability when soil liquefies $\left(r_{u}=1\right)$. The liquefaction state is indicated by the hollow square in Fig. 2. Combining with Fig. 2, the differences between equations (6) and (8) are discussed as below.

(1) The pore pressure accumulation and dissipation phases in Eq. (6) are not distinguished in Eq. (8), due to the numerical character. As to be presented in Section 4.1.3, if $c_{1}$ is large enough (e.g., $c_{1} \geq$ 100 ) and $c_{2}$ is reasonably small (e.g., $c_{2} \leq 2$ ), the further variations of $c_{1}$ and $c_{2}$ will have negligible influences on the numerical results. This is a consequence of the penalty-like treatment by the new dynamic permeability model, as discussed in the end of this subsection.

(2) A controlling point (indicated by hollow circles in Fig. 2) is added as $r_{u}=r_{u}^{c r}$ in Eq. (8), due to the following two reasons. First, the soil permeability represented by DP1-a starts to increase quickly even the excess pore pressure ratio $r_{u}$ is relatively low. This behavior disagrees with the experimental data by Haigh et al. (2012) which showed that the permeability increase is not

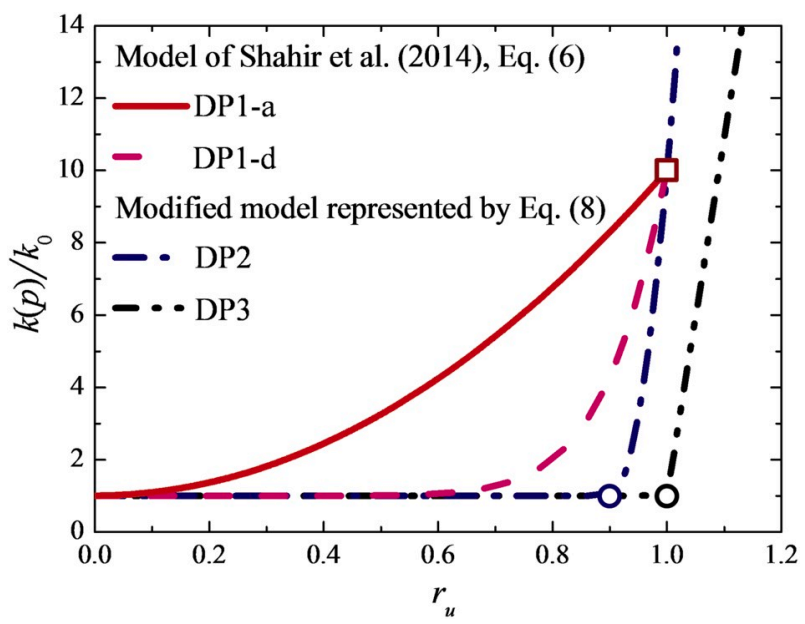

Fig. 2. Variation of soil permeability versus the excess pore pressure ratio. significant unless the effective stress reaches values below 0.1 $\mathrm{kPa}$. An overall increase in permeability is not realistic (Adamidis and Madabhushi, 2016). Therefore, it is reasonable to set a starting value $r_{u}^{\text {cr }}$ to control when the soil permeability begins to increase. Second, experimental results for instantaneous seabed liquefaction (e.g. Liu et al., 2015) provide a basis for the numerical tests using dynamic permeability by trial and error. By conducting such numerical tests, $r_{u}^{\mathrm{cr}}=0.9^{\sim} 1.0$ was found to agree with the experimental data in a reasonable sense. However, $r_{u}^{\mathrm{cr}}=$ 0 (corresponding to Eq. (6)) led to a dramatic deviation with the experimental results, as shown in Section 4.1.1.

(3) The ending point (indicated by the hollow square in Fig. 2) of the $k / k_{0}-r_{u}$ curves in Eq. (6) means that, during liquefaction phase, excess pore pressure ratio cannot exceed the upper limit value of $r_{u}=1.0$. This restriction conforms to the physical understanding that no tensile stress can be sustained in a non-cohesive seabed. However, to numerically implement zero effective stress requirement in liquefied zone, the ending point needs to be removed and the new dynamic permeability model then becomes conceptually similar to the penalty method or perturbed Lagrange method used for nonlinear contact problems (Wang et al., 2019).

With the above three modifications, a new dynamic permeability model expressed by Eq. (8) is proposed to adapt for wave-seabed interaction. In what follows, the values of $r_{u}^{\mathrm{cr}}$ used in DP2 and DP3 are further discussed in detail.

The value of $r_{u}^{\mathrm{cr}}=0.9$ in DP2 means that the seabed has reached a disturbed state before liquefaction. The seabed zone satisfying $r_{u} \geq 0.9$ will have an increased permeability. When the criteria typically requiring $r_{u}=1.0$ is used to determine the liquefaction, it is very likely that no liquefaction can be predicted, as shown in Section 4.1.1. Therefore, it seems necessary to introduce a concept of "quasi-liquefaction" if $r_{u}^{\mathrm{cr}}=0.9$ is used. Under this concept, the liquefaction is not a "yes or no" problem anymore. The liquefaction risk, instead of the liquefaction state, should be used. The liquefaction criteria need modifications accordingly. However, "quasi-liquefaction" is not yet an accepted concept. The results with $r_{u}^{\text {cr }}=0.9$ will be used in Section 4.1.1 only for a tentative discussion. The value of $r_{u}^{\mathrm{cr}}=1$ is recommended in this paper, as adopted by DP3. By using $r_{u}^{\mathrm{cr}}=1$, the permeability will not increase until when the liquefaction state is reached. If no liquefaction occurs, then numerical results by constant and dynamic permeability will be exactly the same. Therefore, the existing liquefaction criteria remain applicable. It can be concluded that, compared with DP2, DP3 minimizes the difficulty of using the new model (Eq. (8)) within the classical computational framework for wave-induced seabed response.

Moreover, another concern about the model parameters is clarified here. At first sight, it seems that $r_{u}^{\mathrm{cr}}=1$ cannot reproduce the permeability increase during the liquefaction state. However, the permeability increase is fulfilled here in an approximate way. As aforementioned, the new model is conceptually similar to the penalty method or perturbed Lagrange method. Therefore, $r_{u}^{\mathrm{cr}}=1$ is approximately satisfied. The residual value $\varepsilon_{r u}=r_{u}-1>0$ can then be used to reproduce the permeability increase according to Eq. (8). At the liquefaction state, the pore pressure is permitted to slightly exceed the initial vertical effective stress of the seabed. Then obviously, $\varepsilon_{r u}$ should be controlled to be close to zero, and this can be achieved by "tuning" the two parameters $c_{1}$ and $c_{2}$. This issue will be further studied in Section 4.1.3.

\subsection{Numerical implementation}

If constant soil permeability is assumed, the weak form and discretization of the boundary value problem (Eq. (1)) by Biot's consolidation theory are standard in soil mechanics. However, once the dynamic permeability is taken into account, the weak form, 
discretization and especially the linearization of the governing equations need to be treated with care. Shahir et al. (2012) implemented their dynamic permeability model by updating the permeability at the end of each time step during seismic analysis. This treatment ignores the nonlinear error correction and is insufficient to correct the tensile stress. Therefore, a nonlinear iterative method is needed to deal with the nonlinearity introduced by dynamic permeability.

Noting that the permeability variation only affects the formulation of mass conservation (Eq. (1b)), the equilibrium equation of the solid-fluid mixture (Eq. (1a)) is still standard and can be found in extensive textbooks. The remaining of this section proceeds to conduct some necessary derivations for the mass conservation equation.

Let $L^{2}(\Omega)$ be the space of real valued square integrable functions defined on the considered seabed domain $\Omega$. $H^{1}(\Omega)$ is used to denote the subspace containing functions having the first order derivatives in $L^{2}(\Omega) . H_{0}^{1}(\Omega)$ is introduced to represent the space of functions in $H^{1}(\Omega)$ which vanish on the boundary $\Gamma$. The weak form of mass conservation equation reads as finding $p \in H_{0}^{1}(\Omega)$ such that there holds:

$\left\langle\frac{\partial \varepsilon_{v}}{\partial t}+n \beta \frac{\partial p}{\partial t}, \varphi\right\rangle+\left\langle\frac{k}{\gamma_{w}} \nabla p, \nabla \varphi\right\rangle=0$

for all $\varphi \in H_{0}^{1}(\Omega)$, with $\langle\cdot, \cdot\rangle$ the inner product on $L^{2}(\Omega)$. The symbol $\langle\cdot, \cdot\rangle$ is introduced here to facilitate the expression. It is also used by List and Radu (2016) to derive the iterative formulation of Richards' equation.

For spatial discretization, the solution space $P_{h}$ and weighting space $V_{h}$ are defined as:

$P_{h}:=\left\{p_{h} \in H^{1}(\Omega) \mid p_{h}=\widehat{p}\right.$ on $\left.\Gamma_{p}\right\}$

$V_{h}:=\left\{v_{h} \in H^{1}(\Omega) \mid v_{h}=0\right.$ on $\left.\Gamma_{p}\right\}$

Then, Eq. (10) can be rewritten as finding $p_{h} \in P_{h}$ such that there holds:

$\left\langle\frac{\partial\left(\varepsilon_{v}\right)_{h}}{\partial t}+n \beta \frac{\partial p_{h}}{\partial t}, v_{h}\right\rangle+\left\langle\frac{k}{\gamma_{w}} \nabla p_{h}, \nabla v_{h}\right\rangle=0$

for all $v_{h} \in V_{h}$, with $\left(\varepsilon_{v}\right)_{h}$ the discrete version of the volumetric strain by using the standard solution space of displacements.

For temporal discretization, the backward Euler method is used. Applying a time integration to Eq. (13), the fully discrete variational formulation is obtained as follows:

$\left\langle\left(\varepsilon_{v}\right)_{h}^{t}-\left(\varepsilon_{v}\right)_{h}^{t-\tau}+n \beta\left(p_{h}^{t}-p_{h}^{t-\tau}\right), v_{h}\right\rangle+\tau\left|\frac{k}{\gamma_{w}} \nabla p_{h}^{t}, \nabla v_{h}\right\rangle=0$

where $\tau$ denotes the time step.

If the inner products $\langle\cdot, \cdot\rangle$ is rewritten as integral forms, then the standard finite element formulation of Biot's equation can be obtained, see Li and Wei (2018) for an instance. However, the symbol $\langle\cdot, \cdot\rangle$ can make the derivations and expressions much more concise.

The Newton-Raphson method is applied to solve Eq. (14). For each nonlinear iteration $j$, the pore pressure solution is denoted by $p_{h}^{t, j}$. Therefore, Eq. (14) is linearized as:

$$
\begin{gathered}
\left\langle\left(\varepsilon_{v}\right)_{h}^{t, j}+n \beta p_{h}^{t, j}, v_{h}\right\rangle+\tau\left|\frac{k^{t, j-1}}{\gamma_{w}} \nabla p_{h}^{t, j}, \nabla v_{h}\right\rangle+\tau\left\langle\frac{\left(k^{\prime}\right)^{t, j-1}}{\gamma_{w}} \nabla p_{h}^{t, j-1} p_{h}^{t, j}, \nabla v_{h}\right\rangle \\
=\left\langle\left(\varepsilon_{v}\right)_{h}^{t-\tau}+n \beta p_{h}^{t-\tau}, v_{h}\right\rangle+\tau\left|\frac{\left(k^{\prime}\right)^{t, j-1}}{\gamma_{w}} \nabla p_{h}^{t, j-1} p_{h}^{t, j-1}, \nabla v_{h}\right\rangle
\end{gathered}
$$

where $k^{\prime}$ denotes the partial derivative of $k$ to $p$. According to Eqs. (8) and (9), $k^{\prime}$ is given by:

$\frac{k^{\prime}(p)}{k_{0}}= \begin{cases}0 & r_{u} \leq r_{u}^{\mathrm{cr}} \\ \left(c_{1}-1\right) c_{2}\left(r_{u}-r_{u}^{\mathrm{cr}}\right)^{c_{2}-1} / \sigma_{v 0}^{\prime} & r_{u}>r_{u}^{\mathrm{cr}}\end{cases}$
Based on Eq. (15) and standard FEM treatments of the equilibrium equation, one can easily obtain the matrix system and iterative procedure used for finding the solution $\left(\mathbf{u}_{h}^{t}, p_{h}^{t}\right)$. The above procedure is numerically implemented in the in-house finite element code (Zhou et al., 2018; Wang et al., 2019), which is to be validated by analytical solutions with constant soil permeability in the next section.

\section{Results and discussions}

\subsection{Comparison with cylinder tests under 1-D wave loading}

Liu et al. (2015) conducted a series of cylinder tests under 1-D wave loading conditions using a $1.8 \mathrm{~m}$ thick sandy deposit. The experimental facility enables installing more pore pressure measurement points (10 points) in the soil layer along the vertical direction than conventional wave flume tests (usually 3 or 4 points). Additional static water pressures were added onto the harmonic dynamic wave pressure, allowing for simulating a greater water depth. These tests provide a good reference to verify the numerical performance and are simulated by the numerical method developed in this paper, using a 2-D mesh shown in Fig. 3. The element size along the $z$ direction is taken as $0.12 \mathrm{~m}$. For the temporal discretization, one wave period is divided into 40 time steps, $i$. e., $\tau=0.025 T$.

Fig. 3 also shows the boundary conditions applied in the simulation. On the bottom and both sides of the seabed, the displacement and fluid flow along the normal direction is constrained as zero. On the seabed surface, the pore pressure of the fluid phase is constrained as a timedependent wave pressure $P_{b}$, and $P_{b}$ is also applied as a uniform distributed pressure to the solid phase. The wave pressure $P_{b}$ is calculated by the linear wave theory:

$P_{b}=p_{0} \cos (\omega t)$

$p_{0}=\frac{\gamma_{w} H}{2 \cosh (\lambda h)}, \quad \omega=2 \pi / T, \quad \lambda=2 \pi / L$

with the pressure amplitude $p_{0}$ on the seabed surface, the wave angular frequency $\omega$, wave period $T$, wave height $H$, water depth $h$, wave number $\lambda$ and wave length $L$. The wave length $L$ is iteratively computed from the wave dispersion relation:

$L=\frac{g T^{2}}{2 \pi} \tanh \left(\frac{2 \pi}{L} h\right)$

Linear elasticity behavior is assumed to the solid phase with material

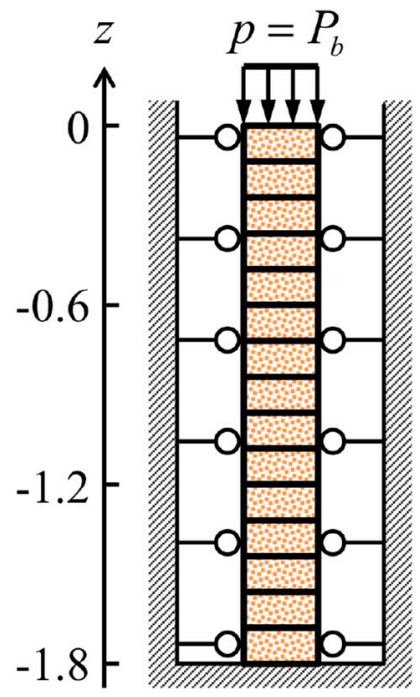

Fig. 3. Computational mesh and boundary conditions of the 1-D cylinder tests. 
parameters given by: shear modulus $G=1.27 \times 10^{7}$ Pa, Poisson's ratio $\nu=0.3$. The initial value of the sand permeability is given as $k_{0}=1.8 \times$ $10^{-4} \mathrm{~m} / \mathrm{s}$. The water depth $h$ is $5.2 \mathrm{~m}$, taking into account the additional static water pressure added in the tests. Liu et al. (2015) reported 24 experimental tests in total, by changing the soil porosity $n$, soil saturation $S_{r}$, wave period $T$ and pressure amplitude $p_{0}$. The "Test 20", see Table 2 in (Liu et al., 2015), is used in Section 4.1.1 to evaluate the performance of dynamic permeability models. Then Section 4.1.2 conducts a comparison with 12 experimental tests under various conditions. The effects of model parameters and the nonlinear convergence performance are discussed in Section 4.1.3 and Section 4.1.4, respectively.

\subsubsection{Performance of dynamic permeability models: case study}

For "Test 20" in Liu et al. (2015), the computational parameters are given by: $n=0.425, \gamma^{\prime}=9.41 \mathrm{kN} / \mathrm{m}^{3}, S_{r}=0.996, T=9 \mathrm{~s}$ and $p_{0}=$ $34.32 \mathrm{kPa}$. In this section, four numerical tests are conducted, with one using constant permeability and the others using dynamic permeability, as listed in Table 1.

Fig. 4 presents the variation of the pressure amplitude along with the soil depth. As shown in Fig. 4, the numerical result using constant permeability (CP) achieves a good agreement with the analytical solution by Hsu and Jeng (1994), validating the numerical code developed in this paper. If the model parameters in Shahir et al. (2014), i.e. DP1-a, are directly used here, a dramatic deviation can be observed between the numerical and experimental results. This implies that an overall increase in the soil permeability is inappropriate for simulating the wave-induced seabed response. In contrast, the cases using constant permeability (CP), DP2 and DP3 all coincide with the experimental data in a reasonable sense. The permeability variations in DP2 and DP3 result in a decrease in the pressure amplitude, but the decrease is not significant.

For wave-induced seabed response, the phase lag is a key character and is found here to have a considerable influence on the liquefaction depth. The two cases CP and DP3 are discussed as below. The discussions for DP2 and DP1-a are similar. Figs. 5-7 shows the phase lag effect on the temporal variation of $p, p-P_{b}$ and $\sigma_{v 0}^{\prime}-\left(p-P_{b}\right)$ at different soil depths. It can be seen from Fig. 5 that the trough values of $p$ occur when $t \geq 0.5 T$. The trough values occur later in deeper position, well corroborated by the general characteristics of wave-induced seabed response. Fig. 6 shows that the peak values of $p-P_{b}$ also occur later in deeper position, but all the peak values occur when $t \leq 0.5 T$. This can then result in an interesting phenomenon that the liquefaction occurs before the instant of $t=0.5 T$, and the maximum liquefaction depth appears prior to wave trough, as shown in Fig. 7.

This phenomenon can be observed more apparently in Fig. 8 by plotting the vertical distribution of $\sigma_{v 0}^{\prime}-\left(p-P_{b}\right)$ at two representative instants, i.e., $t=0.45 T$ and $t=0.5 T$. The following criterion by Zen and Yamazaki (1990) is hereafter used to determine the wave-induced instantaneous liquefaction:

$p-P_{b} \geq \sigma_{v 0}^{\prime}$

The liquefaction depths at $t=0.5 T$ are $0.85 \mathrm{~m}$ and $0.46 \mathrm{~m}$ for $\mathrm{CP}$ and DP3, respectively. However, the instant of $t=0.45 T$ is found to have the maximum liquefaction depths of $1.02 \mathrm{~m}$ and $0.67 \mathrm{~m}$ for CP and DP3 respectively. It can be concluded that simply investigating the instant when the wave trough arrives $(t=0.5 T)$ is not enough. Instead, the temporal variation should be considered. It is also notable that the phase

Table 1

Numerical tests for “Test 20" in Liu et al. (2015).

\begin{tabular}{lllll}
\hline Case abbreviation & $c_{1}$ & $c_{2}$ & $r_{u}^{\text {rr }}$ & Remarks \\
\hline CP & - & - & - & Constant permeability \\
DP1-a & 10 & 2 & 0 & Dynamic permeability \\
DP2 & 901 & 2 & 0.9 & \\
DP3 & 100 & 1 & 1 & \\
\hline
\end{tabular}

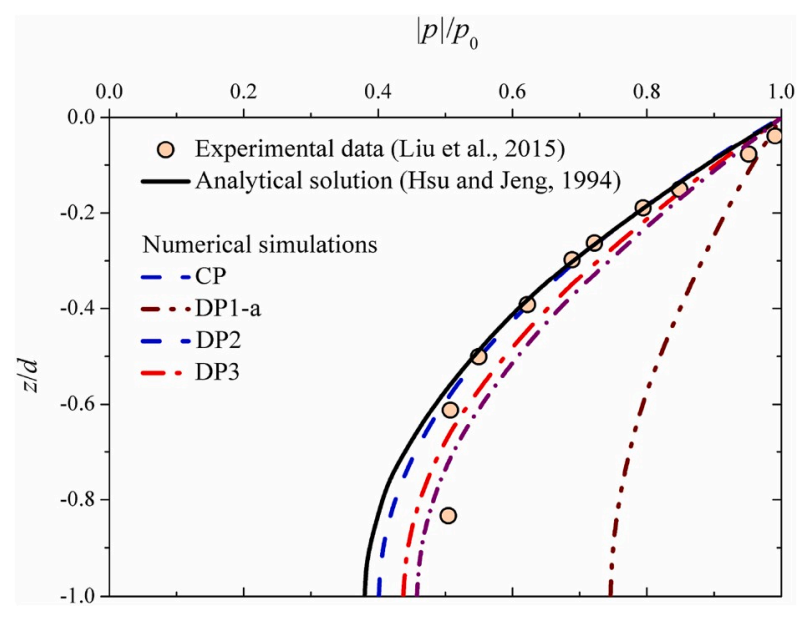

Fig. 4. The pressure amplitude versus the soil depth.

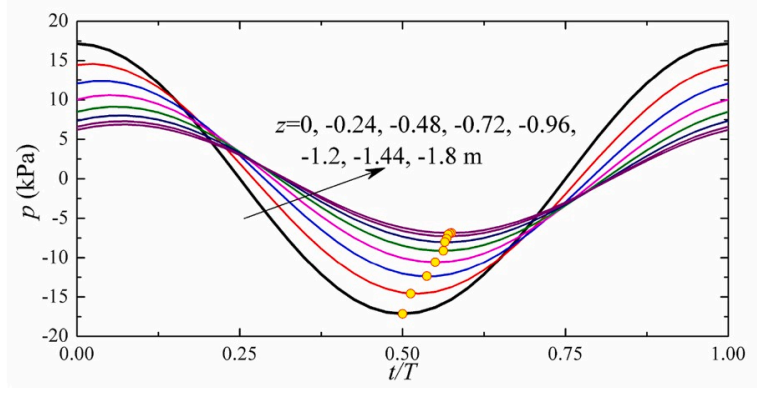

(a) $\mathrm{CP}$

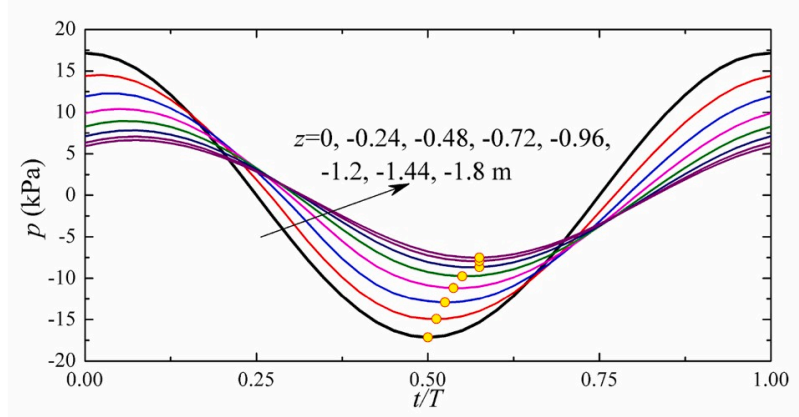

(b) DP3

Fig. 5. The temporal variation of $p$ at different soil depths.

lag would not always cause the "early liquefaction" phenomenon, as further discussed in Section 4.1.2 (refer to Fig. 14).

For all the four cases (CP, DP2, DP3 and DP1-a), Fig. 9 gives the vertical distributions of $p-P_{b}$ and $\sigma_{v 0}^{\prime}-\left(p-P_{b}\right)$ when maximum liquefaction depth is observed $(t=0.45 T)$. In Fig. 9(a), the waveinduced pore pressure by constant permeability (CP) exceeds the initial vertical effective stress (i.e., $r_{u}>1$ ) in the liquefaction zone. This leads to tensile stresses observed in Fig. 9(b), which should never appear in a non-cohesive sandy seabed. In contrast, DP2 and DP3 remarkably ease this nonphysical behavior. However, no liquefaction is observed in DP2. Noting that $r_{u}^{\mathrm{cr}}=0.9$ is used in DP2, the criterion by Eq. (19) is tentatively modified here as $p-P_{b} \geq 0.9 \sigma_{v 0}^{\prime}$ to determine a zone with high liquefaction risks instead of liquefaction state. The corresponding depth is determined as $0.85 \mathrm{~m}$. It is notable that this type of modification 


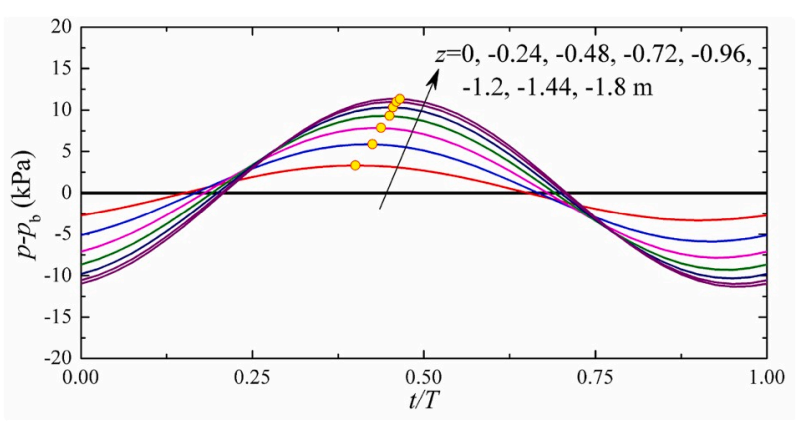

(a) $\mathrm{CP}$

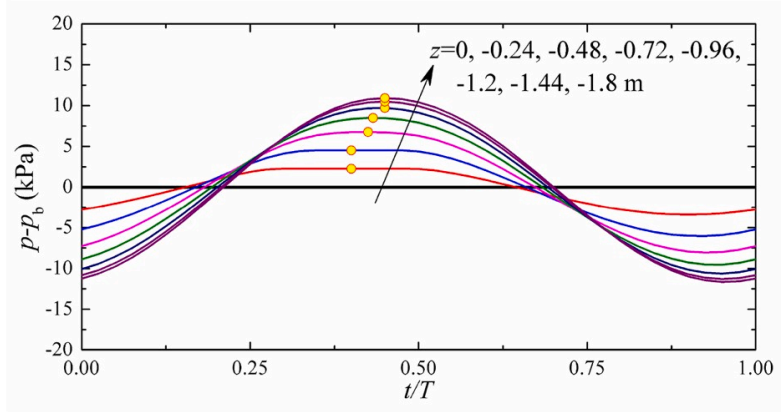

(b) DP3

Fig. 6. The temporal variation of $p-P_{b}$ at different soil depths.

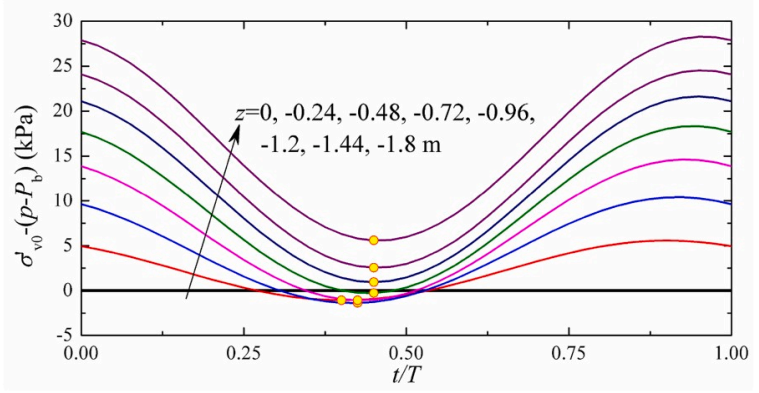

(a) $\mathrm{CP}$

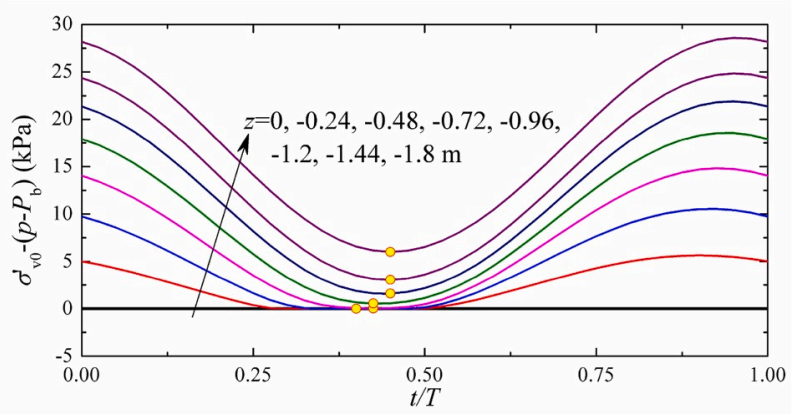

(b) DP3

Fig. 7. Temporal variation of $\sigma_{v 0}^{\prime}-\left(p-P_{b}\right)$ at different soil depths.

cannot work for DP1-a because $r_{u}^{\mathrm{cr}}=0$ is used in this case.

Based on the above observations, it was found that DP1-a is not suitable for simulating wave-induced seabed response, while DP2 leads to some additional difficulties in determining the liquefaction zone. Therefore, the results by DP2 and DP1-a will not be discussed hereafter. Instead, DP3 is recommended when dynamic permeability is needed.

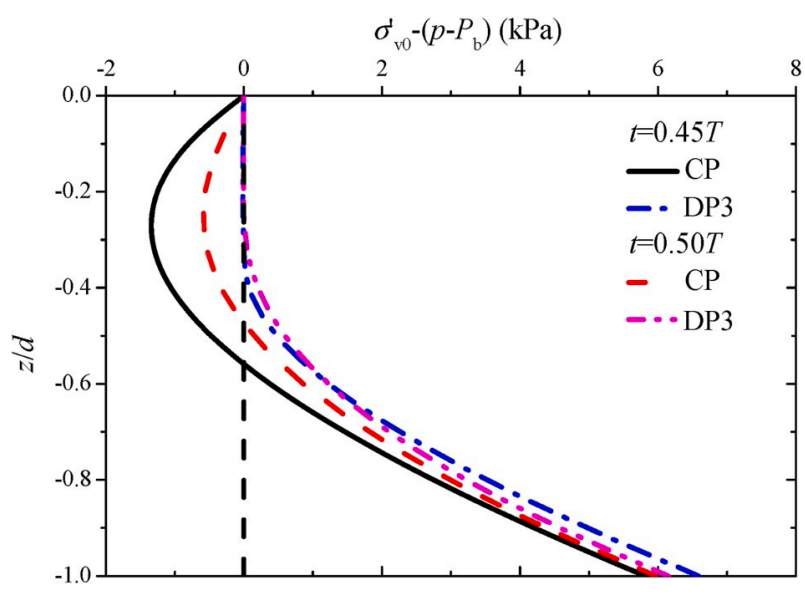

Fig. 8. Vertical distribution of $\sigma_{v 0}^{\prime}-\left(p-P_{b}\right)$ at two different instants.

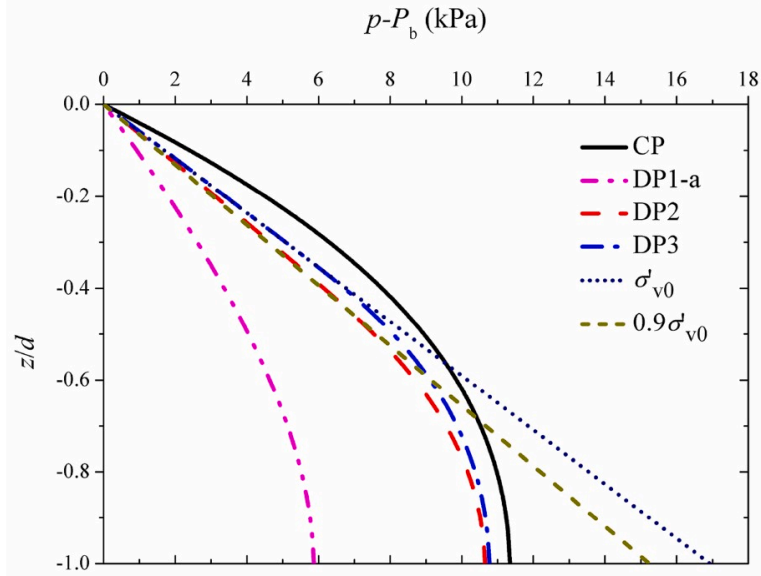

(a)

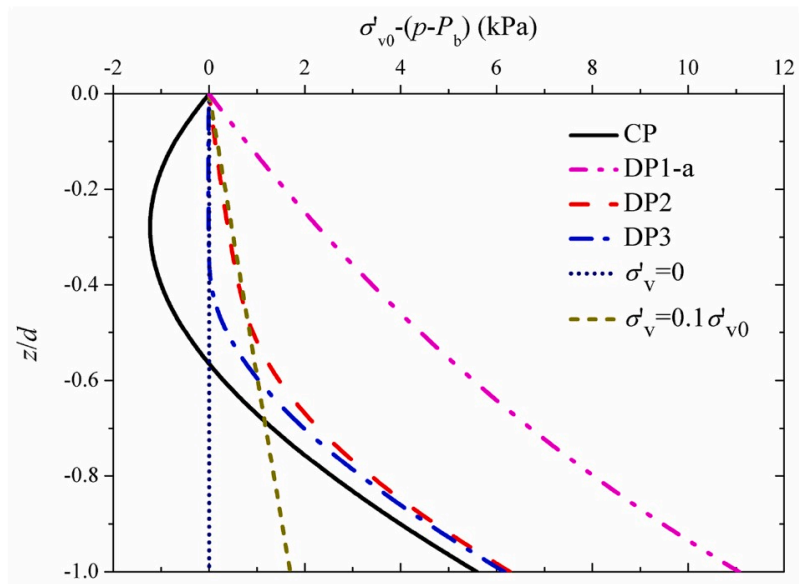

(b)

Fig. 9. Vertical distributions of (a) $p-P_{b}$; and (b) $\sigma_{v 0}^{\prime}-\left(p-P_{b}\right)$.

4.1.2. Comparison with cylinder tests using proposed dynamic permeability model

The above discussions show that the dynamic permeability model proposed in this paper can improve the numerical performance. In order to further validate the improvement, this section conducts a comparison with more experimental tests, shown in Figs. 10-13. Three values of wave pressure amplitude $p_{0}$ are considered, i.e., $12.10 \mathrm{kPa}, 24.52 \mathrm{kPa}$ 
and $34.32 \mathrm{kPa}$.

In Figs. 10(a)-11(a), all the numerical results generally coincide with the experimental data in a reasonable sense. It seems that the improvement of using dynamic permeability is not significant for sands with high saturation $\left(S_{r}=0.996\right)$. This is mainly due to the fact that the tensile stress in case CP is under a relatively low magnitude, as showed by Figs. 10(b)-11(b). Therefore, the difference between constant and dynamic permeability is not apparent in the vertical distribution of the pressure amplitude $|p|$. For the experimental tests under $12.10 \mathrm{kPa}$ and $24.52 \mathrm{kPa}$ (i.e., tests $22-23$ in Fig. 12 and tests $16-17$ in Fig. 13), the numerical results by CP and DP3 even stay almost the same.

However, the numerical performance for sands with low saturation $\left(S_{r}=0.951\right)$ can be greatly improved by using the dynamic permeability model proposed in this paper. As shown in Figs. 12(a) and 13(a), the conventional model using constant permeability (CP) leads to a dramatic discrepancy with the experimental results. This is a consequence of the abnormally large negative values (tensile stresses) shown in Figs. 12(b) and 13(b) (close to $-11 \mathrm{kPa}$ in Test 2). These abnormal tensile stresses are greatly eased by adopting DP3. Meanwhile, DP3 obtains acceptable agreements with the experimental data, especially the overall experimental tendency is well reproduced. Discrepancy between the numerical and experimental results mainly appears near the seabed surface. The discrepancy can be primarily attributed to the

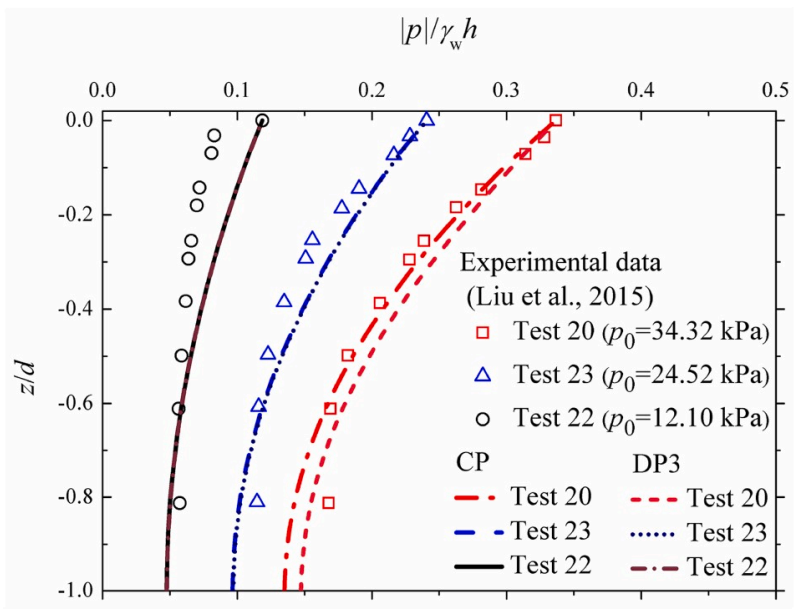

(a)

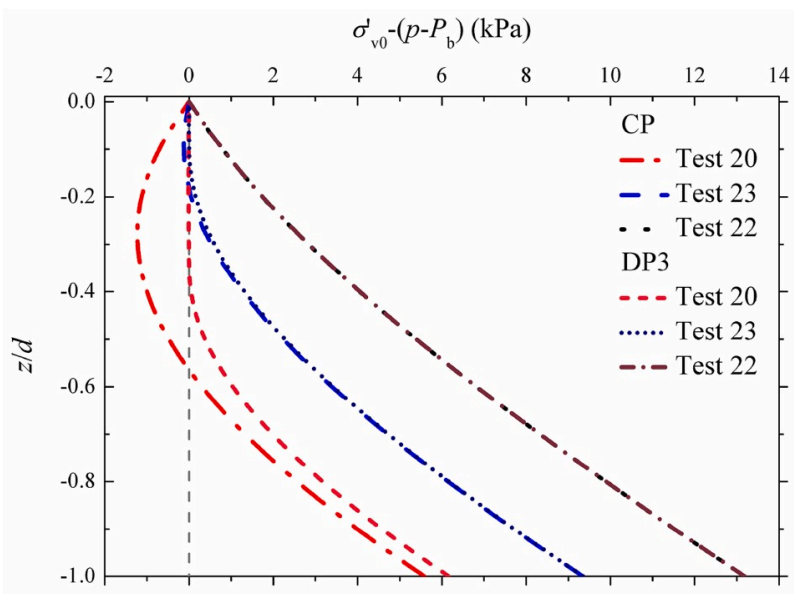

(b)

Fig. 10. Vertical distributions of (a) pressure amplitude $|p|$; and (b) $\sigma_{v 0}^{\prime}-(p-$ $P_{b}$ ). (Loose sand with high saturation: $n=0.425, \gamma^{\prime}=9.41 \mathrm{kN} / \mathrm{m}^{3}, S_{r}=$ 0.996).

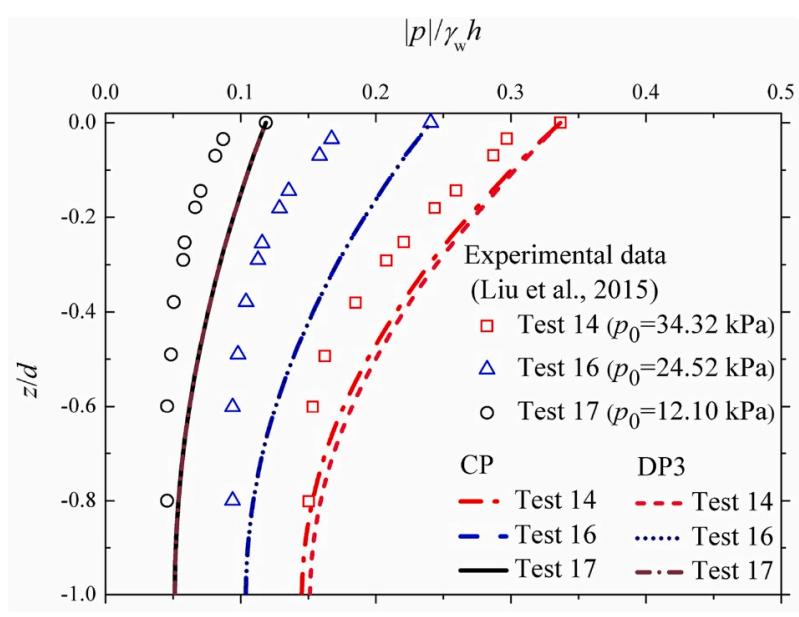

(a)

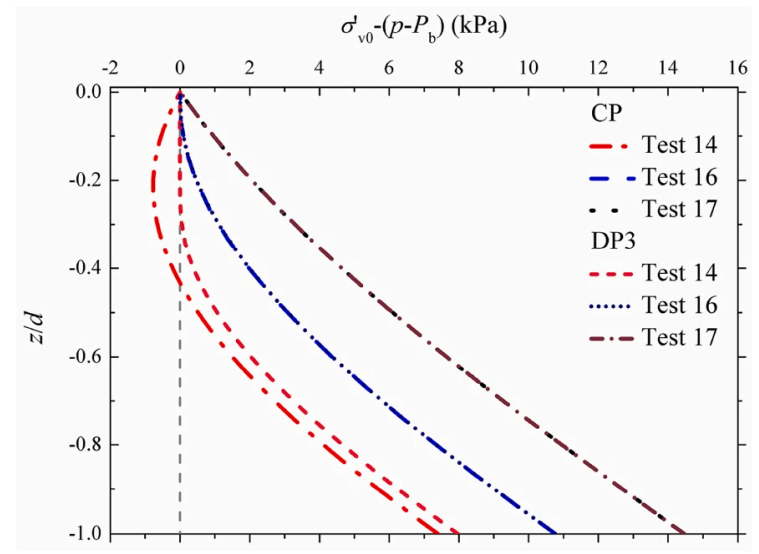

(b)

Fig. 11. Vertical distributions of (a) pressure amplitude $|p|$; and (b) $\sigma_{v 0}^{\prime}-(p-$ $P_{b}$ ). (Dense sand with high saturation: $n=0.387, \gamma^{\prime}=10.0 \mathrm{kN} / \mathrm{m}^{3}, S_{r}=$ 0.996).

dynamic movement pattern of the soil particles near the sample surface. It is not surprise that this type of dynamic behaviors in the liquefied zone cannot be reproduced by quasi-static numerical simulations under the assumption of porous medium. Nevertheless, the dynamic permeability model provides a solid improvement to existing computational framework in a convenient way.

For all the above tests, the maximum liquefaction depths predicted by CP and DP3 are given in Table 2. The last column in Table 2 is the ratio of the liquefaction depth by CP to the value by DP3. The symbol "-." means that no liquefaction is found. It can be seen that the liquefaction depth by CP is $1.52-2.00$ times the value by DP3. The dynamic permeability has a significant influence on the liquefaction estimation.

In Figs. 10-13, the distributions of $\sigma_{v 0}^{\prime}-\left(p-P_{b}\right)$ correspond to the instant when the negative peak value of $\sigma_{v 0}^{\prime}-\left(p-P_{b}\right)$ occurs or the maximum liquefaction depth emerges (if there is any liquefaction). Again, the phase lag effect of causing the "early liquefaction" phenomenon is considered. Here the "Test 2" (low saturation) is discussed by observing four instants. As shown in Fig. 14, the maximum liquefaction depth occurs when the wave trough arrives $(t=0.5 T)$. This observation implies that the "early liquefaction" phenomenon does not always appear, but depends on the computational parameters.

\subsubsection{Effects of model parameters}

As can be seen from Section 4.1.2, when constant permeability is used, "Test 2" has much larger tensile stresses than other tests. 
(a)

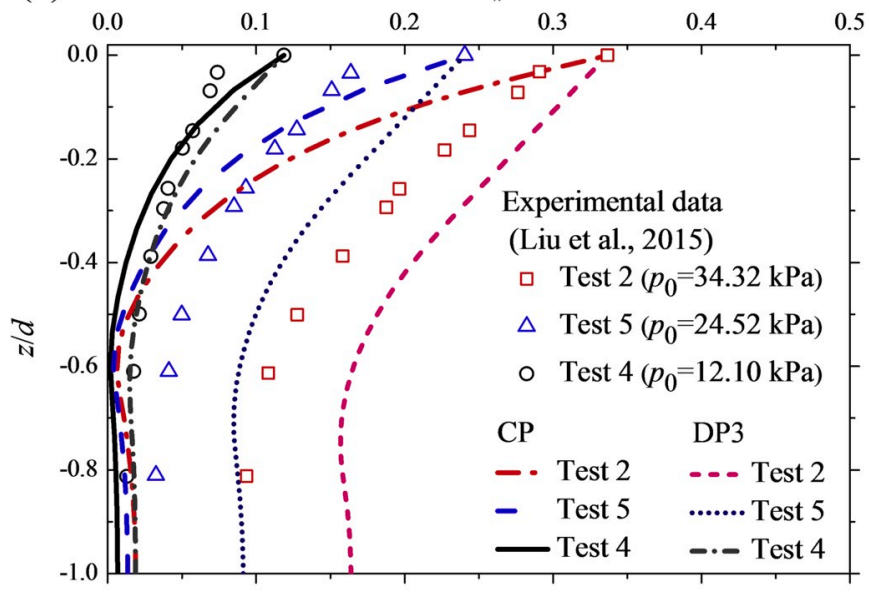

(b)

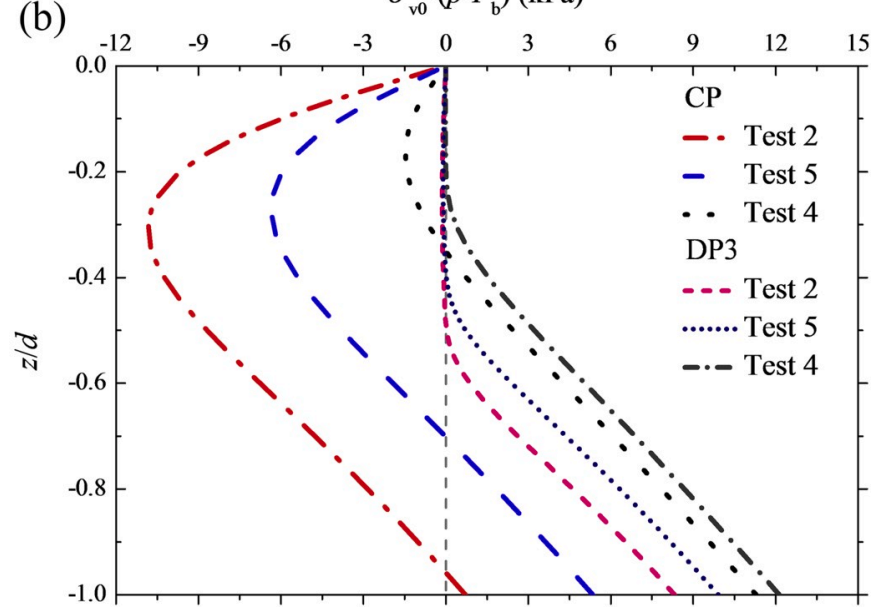

Fig. 12. Vertical distributions of (a) pressure amplitude $|p|$; and (b) $\sigma_{v 0}^{\prime}-(p-$ $P_{b}$ ). (Loose sand with low saturation: $n=0.425, \gamma^{\prime}=9.41 \mathrm{kN} / \mathrm{m}^{3}, S_{r}=$ 0.951).

Therefore, "Test 2" is chosen here to investigate the effects of model parameters by adopting four groups of $c_{1}$ and $c_{2}$ while keeping $r_{u}^{\text {cr }}=1$.

First, the model parameter $c_{2}=1$ is fixed. By using $c_{1}=10,100$ and 1000 , the minimum values of $\sigma_{v 0}^{\prime}-\left(p-P_{b}\right)$ are reduced to -1.546 , -0.135 and $-0.012 \mathrm{kPa}$, respectively. With the increase of $c_{1}$, the tensile stresses approach zero gradually. If $c_{1}=10$ is used, apparent tensile stresses can still occur, as shown in Fig. 15(a). Nevertheless, it already obtains a considerable improvement over $\mathrm{CP}$. The results by $c_{1}=100$ and 1000 are almost the same and the tensile stresses become unapparent. These results imply that $c_{1}=100$ is large enough to obtain a good numerical performance.

Then, the model parameter $=100$ is fixed. Two values of $c_{2}=1$ and 2 are compared. The $k / k_{0}-r_{u}$ curves are shown in Fig. 16 (a), according to Eq. (8). It can be seen that $c_{2}=1$ has a shaper increase than $c_{2}=2$ until $r_{u}=2$. Fig. 15(b) already showed that the maximum values of $r_{u}$ obtained by dynamic permeability never exceed 2 . This is to say, $c_{2}=1$ can provide a larger permeability increase to correct the tensile stress during the nonlinear iterative procedure. Therefore, $c_{2}=1$ can obtain a better performance than $c_{2}=2$, as shown in Fig. 15(a).

Fig. 16 (b) further shows the vertical distribution of the soil permeability. It can be seen that the shallow position of the seabed has the maximum permeability when using dynamic permeability. The reason is that $r_{u}$ is rather large by constant permeability (CP) at the shallow position (see Fig. 15(b)), where greater permeability is needed to correct

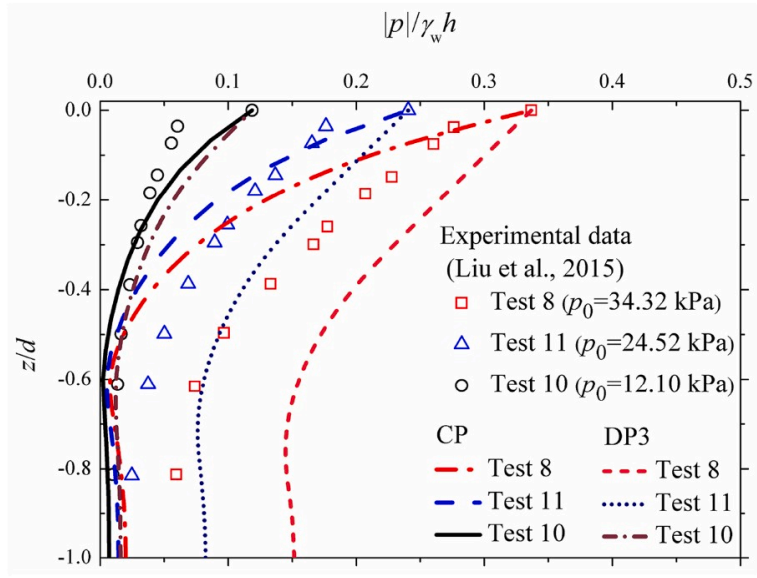

(a)

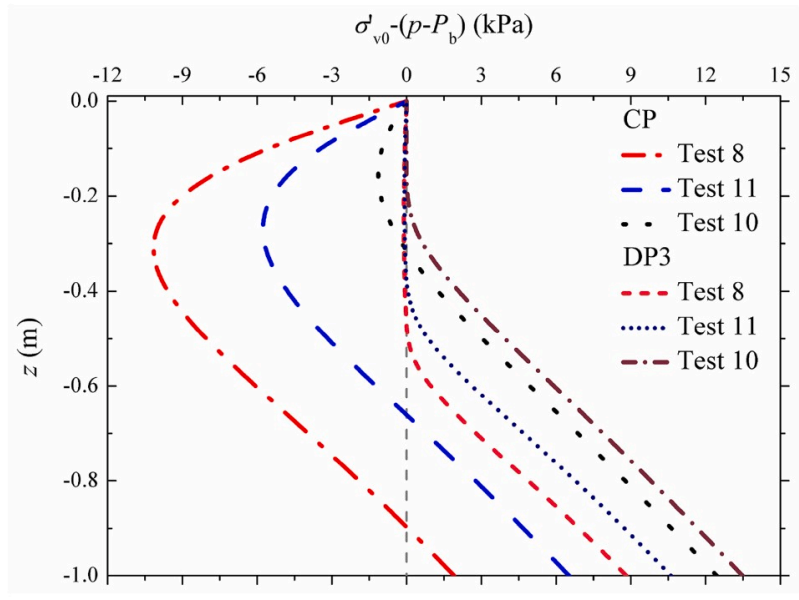

(b)

Fig. 13. Vertical distributions of (a) pressure amplitude $|p|$; and (b) $\sigma_{v 0}^{\prime}-(p-$ $P_{b}$ ). (Dense sand with low saturation: $n=0.387, \gamma^{\prime}=10.0 \mathrm{kN} / \mathrm{m}^{3}, S_{r}=$ 0.951).

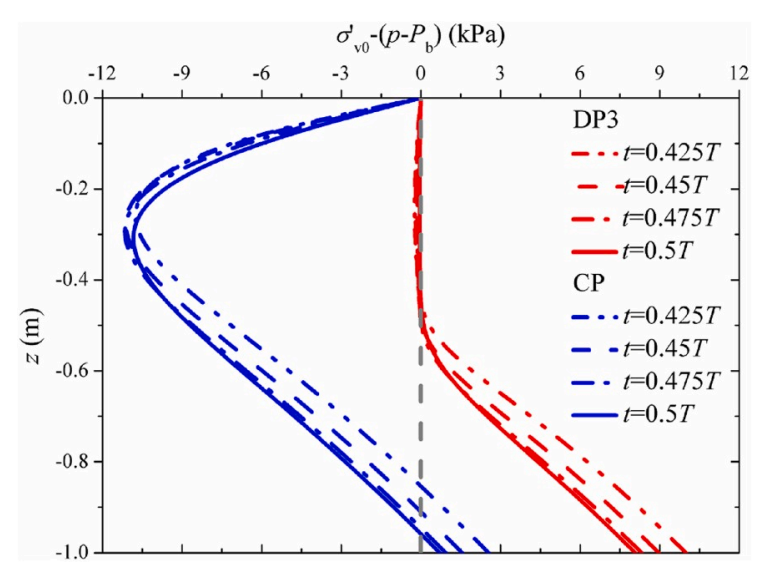

Fig. 14. Vertical distribution of $\sigma_{v 0}^{\prime}-\left(p-P_{b}\right)$ at four different instants in "Test 2".

the unreasonable behavior of $r_{u}>1$. Once the correction is accomplished by the iterative nonlinear procedure, all the four parameter groups are found to have a similar permeability distribution. It is more interesting that the two values of $c_{1}=100$ and 1000 have almost the same permeability. This implies that the increase of $c_{1}$ will give a 
Table 2

Maximum liquefaction depths predicted by different models.

\begin{tabular}{|c|c|c|c|c|c|c|c|c|}
\hline \multirow[t]{2}{*}{ Remarks } & \multirow[t]{2}{*}{ Test no. } & \multirow[t]{2}{*}{$p_{0}(\mathrm{kPa})$} & \multirow[t]{2}{*}{$n$} & \multirow[t]{2}{*}{$\gamma^{\prime}\left(\mathrm{kN} / \mathrm{m}^{3}\right)$} & \multirow[t]{2}{*}{$S_{r}$} & \multicolumn{2}{|c|}{ Liquefaction depth (m) } & \multirow[t]{2}{*}{ ratio } \\
\hline & & & & & & $\mathrm{CP}$ & DP3 & \\
\hline \multirow[t]{3}{*}{ Loose sand with high saturation } & 20 & 34.32 & \multirow[t]{3}{*}{0.425} & \multirow{3}{*}{9.41} & \multirow[t]{3}{*}{0.996} & 1.02 & 0.67 & 1.53 \\
\hline & 23 & 24.52 & & & & 0.33 & 0.21 & 1.60 \\
\hline & 22 & 12.10 & & & & - & - & - \\
\hline \multirow[t]{3}{*}{ Dense sand with high saturation } & 14 & 34.32 & \multirow[t]{3}{*}{0.387} & \multirow[t]{3}{*}{10.0} & \multirow[t]{3}{*}{0.996} & 0.78 & 0.50 & 1.56 \\
\hline & 17 & 24.52 & & & & - & - & - \\
\hline & 16 & 12.10 & & & & - & - & - \\
\hline \multirow[t]{3}{*}{ Loose sand with low saturation } & 2 & 34.32 & \multirow[t]{3}{*}{0.425} & \multirow[t]{3}{*}{9.41} & \multirow[t]{3}{*}{0.951} & 1.73 & 0.87 & 2.00 \\
\hline & 5 & 24.52 & & & & 1.27 & 0.75 & 1.70 \\
\hline & 4 & 12.10 & & & & 0.63 & 0.38 & 1.67 \\
\hline \multirow[t]{3}{*}{ Dense sand with low saturation } & 8 & 34.32 & \multirow[t]{3}{*}{0.387} & \multirow[t]{3}{*}{10.0} & \multirow[t]{3}{*}{0.951} & 1.62 & 0.86 & 1.89 \\
\hline & 11 & 24.52 & & & & 1.19 & 0.74 & 1.62 \\
\hline & 10 & 12.10 & & & & 0.57 & 0.36 & 1.57 \\
\hline
\end{tabular}

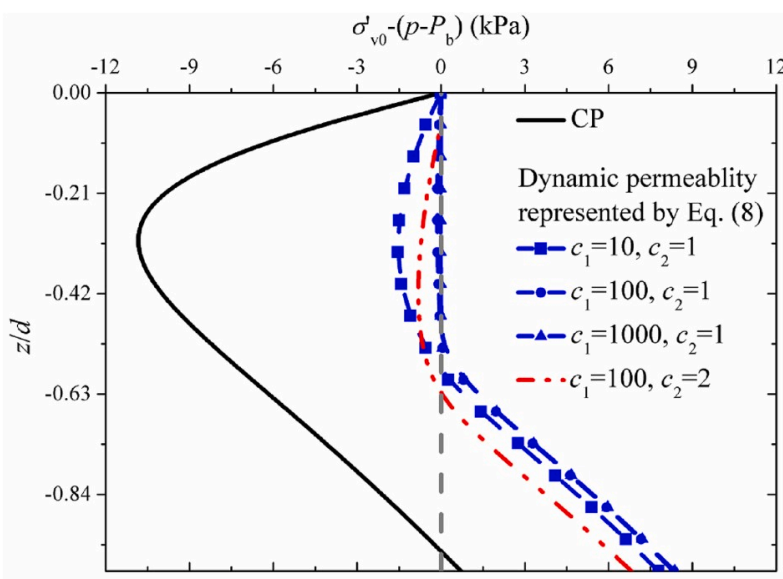

(a)

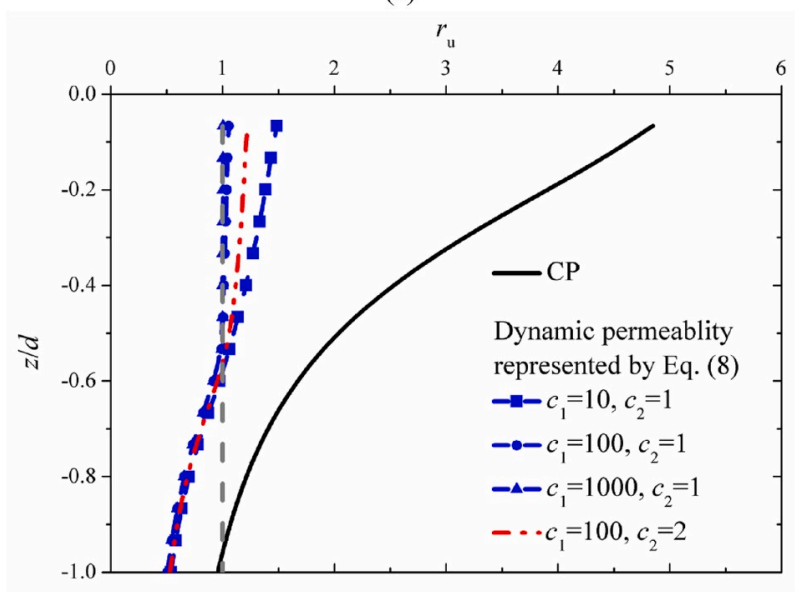

(b)

Fig. 15. Vertical distributions of (a) $\sigma_{v 0}^{\prime}-\left(p-P_{b}\right)$; and (b) $r_{u}$.

converged result, making the dynamic permeability model somehow a parameter-free treatment. More precisely, once $c_{1}$ is large enough (i.e., 100 ), the further variation of $c_{1}$ will have a negligible influence on the numerical result. This performance is similar to the penalty method within the constrained variational principle (Zhou et al., 2018; Wang et al., 2019). However, it is also notable that the dynamic permeability treatment here is not really a penalty method. This may cause some difficulties for the nonlinear iterative method to achieve a convergence, as discussed as below.

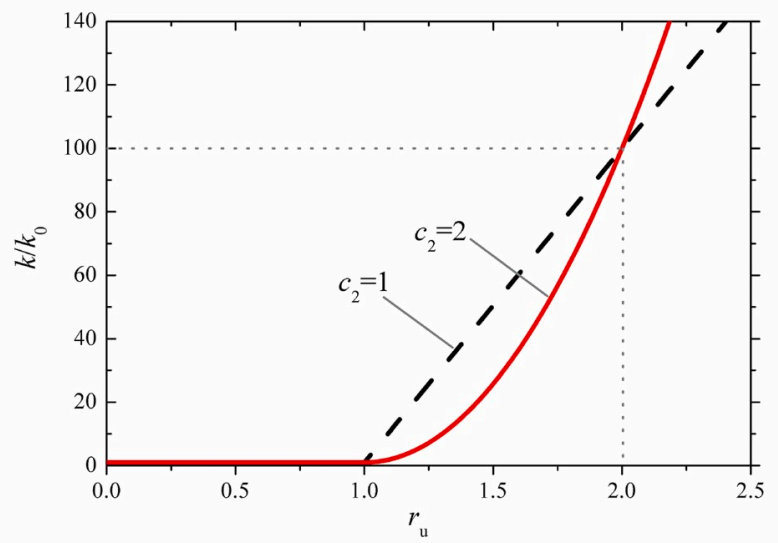

(a)

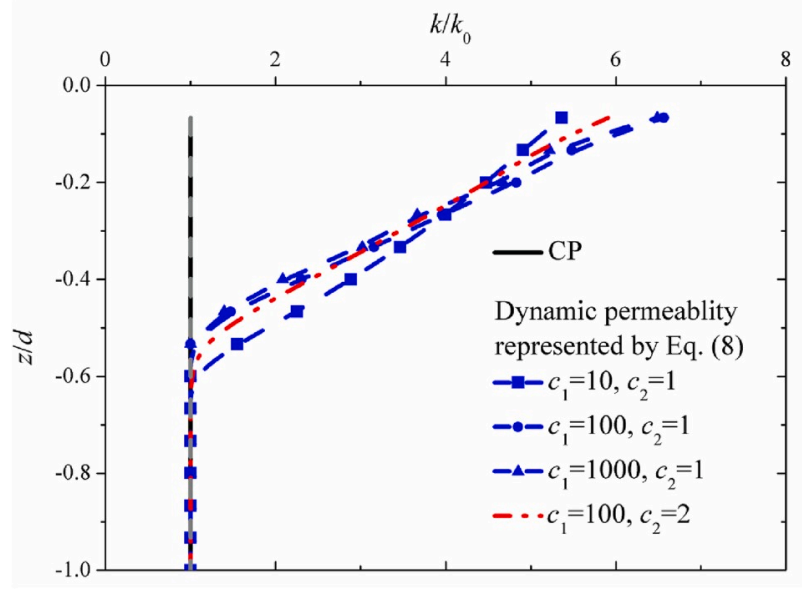

(b)

Fig. 16. Parametric study: (a) $k / k_{0}-r_{u}$ curves by using $c_{2}=1$ and 2 ; (b) the vertical distribution of soil permeability $k / k_{0}$.

\subsubsection{Nonlinear convergence performance}

For the case using DP3 to simulate "Test 2", the convergence performance of the nonlinear iterative method is discussed in this section. Fig. 17(a) gives the iteration numbers cost by each time step. The liquefaction depth is also shown in Fig. 17(a) to provide a reference. It is observed that the iteration number generally increases along with the liquefaction depth. When the liquefaction occurs, the Newton-Raphson method requires averagely more than 10 iterations to achieve the convergence. For two typical time steps marked in Fig. 17(a), the 


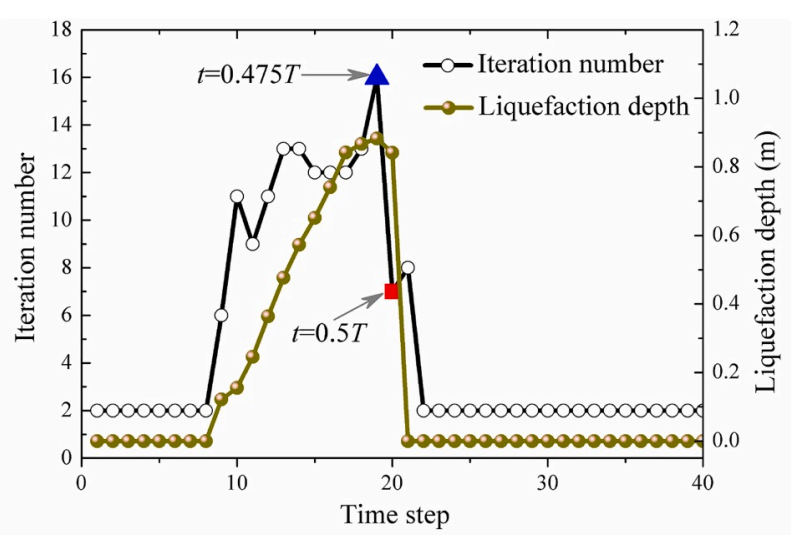

(a)

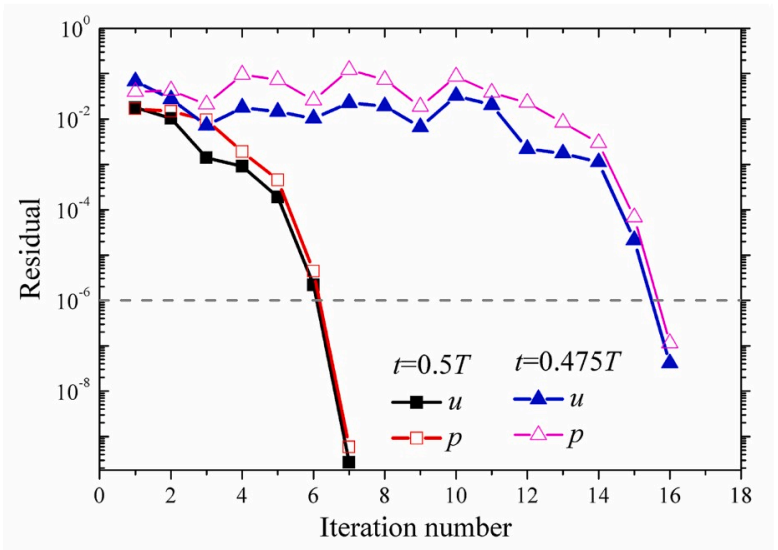

(b)

Fig. 17. Convergence performance: (a) iteration number and liquefaction depth versus time step; (b) residual versus iteration number.

convergence process is investigated in Fig. 17(b), wherein the residual is defined as the ratio of the incremental solution to the total solution. If the residuals of displacement $u$ and pore pressure $p$ are both smaller than $1 \times 10^{-6}$, the iterative procedure is regarded as converged. Theoretically, the Newton-Raphson method should be quadratically convergent. The performance shown in Fig. 17(b) cannot be regarded as good.

For the time step of $t=0.475 T$ which needs the maximum iterations, Fig. 18 further shows the vertical distributions of $\sigma_{v 0}^{\prime}-\left(p-P_{b}\right)$ and $r_{u}$ during the iterative process. Serious numerical oscillations are observed during the first several iterations. It takes plenty of subsequent iterations to remove these oscillations. The reason of causing these oscillations during the iterative process may originate from the Darcy's law, wherein the permeability affects the pore pressure. Once the soil permeability updates, the pore pressure changes accordingly. The convergence is achieved by seeking a compulsory agreement of permeability variation and pore pressure variation. However, the pore pressure changes during the iterations without control by the "inflection point" of $r_{u}=r_{u}^{\mathrm{cr}}$ in the $k / k_{0}-r_{u}$ curve. The convergence cannot be expected if the $k / k_{0}-r_{u}$ curve is extremely sharp. What is worse is that numerical divergences can even occur in 2-D simulations, as to be demonstrated in the next section.

\subsection{Application of dynamic permeability in 2-D wave-seabed interactions}

\subsubsection{Computational model and parameters}

Fig. 19 shows the computational mesh, wherein the shallow layer and the $x$-directional central part have smaller element sizes. This setup aims to provide a high numerical accuracy for the potential liquefaction

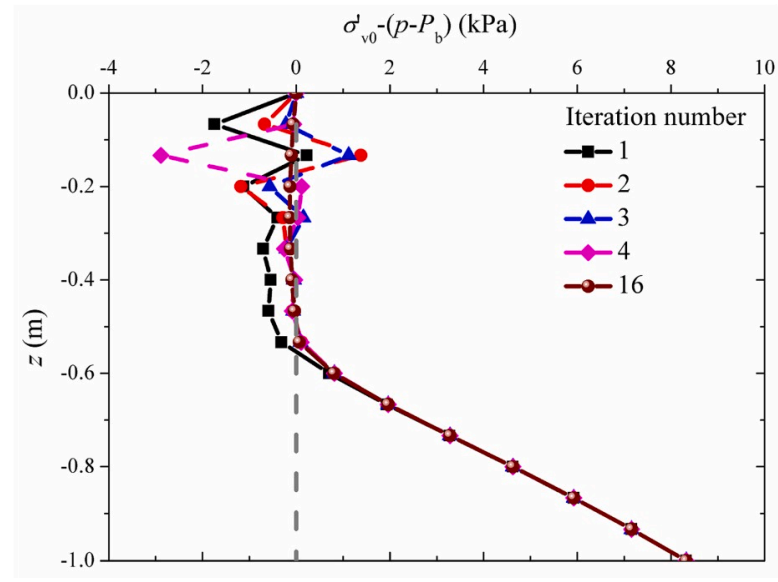

(a)

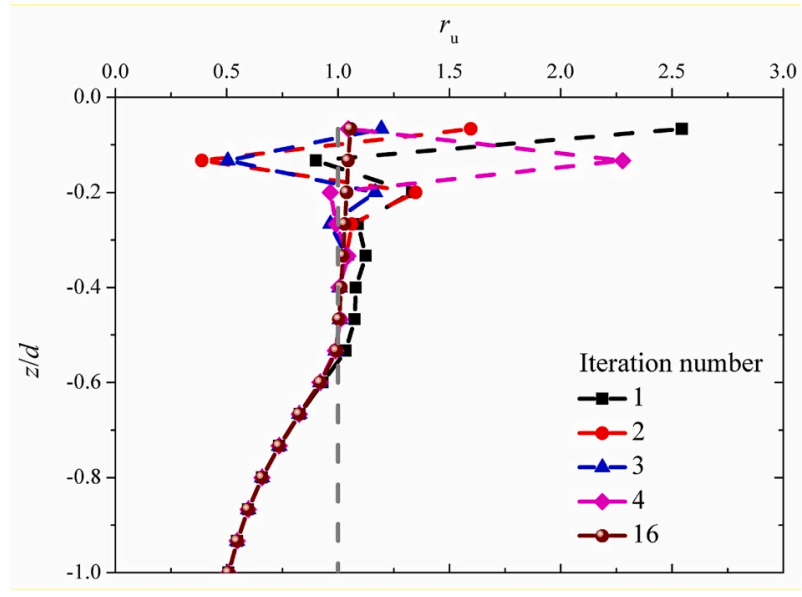

(b)

Fig. 18. Vertical distributions during the iterative process: (a) $\sigma_{v 0}^{\prime}-\left(p-P_{b}\right)$; and (b) $r_{u}$.

zone. The wave parameters and soil properties are also provided in Fig. 19. To simulate the infinite-thick seabed, the seabed thickness should be larger than the wave length $L(\approx 61.4 \mathrm{~m}$ here), as reported by Hsu and Jeng (1994). Here, the seabed thickness is taken as $100 \mathrm{~m}$. The seabed length is set as three times of the wave length. As reported in Ye and Jeng (2012), such a computational domain is sufficient for the concerned region at the $x$-directional central part. The boundary conditions are similar to those in Fig. 3. The only difference is that the wave pressure $P_{b}$ here is a function of both $x$-axis and time $t$ :

$P_{b}=p_{0} \cos (\lambda x-\omega t)$

In the previous section, the 1-D cylinder tests are simulated by several model parameters of Eq. (8). Although the convergence rate is not satisfactory, the nonlinear iterative procedure obtained converged results for all the tested numerical simulations, even for the value of $c_{1}$ $=1000$ which introduces a very strong nonlinearity. This consequence mainly benefits from the relatively simple behavior in 1-D cylinder tests. However, the 2-D simulation is much more complicated due to the horizontal interaction in the porous-medium seabed. The two cases DP2 and DP3 in Table 1 were both found leading to numerical instability or divergence, as shown in Section 4.2.3.

The reason of causing this type of strong nonlinearity can be traced back to the Darcy's law. Stable and robust treatments are not readily available. The undergoing work shows that the Darcy's law may need to be revisited and profoundly discussed. However, this paper focuses on dealing with the Darcy's law with dynamic permeability. Therefore, to 


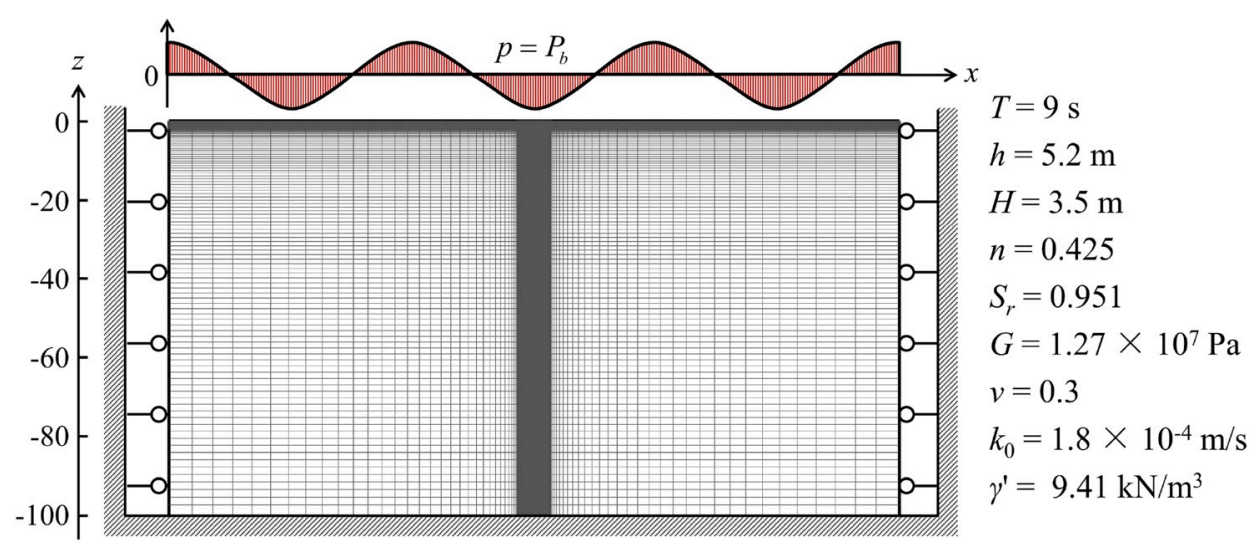

Fig. 19. Computational mesh and boundary conditions of the 2-D simulation.

obtain a convergence here in this section, the parameters $c_{1}=10, c_{2}=$ 1 and $r_{u}^{\mathrm{cr}}=1$, milder than DP2 and DP3, are used for the simulation of 2D wave-seabed interactions.

\subsubsection{Numerical results}

In the simulation here, the "early liquefaction" phenomenon caused by the phase lag is not obvious. This is to say, the maximum liquefaction depth can be expected under the wave trough. As the $x$-directional central part is the concerned region, all the numerical results hereafter are then given at the instant of $t=T$ when the position of $x=1.5 \mathrm{~L}$ is under wave trough. Fig. 20 shows the contours of $p-P_{b}$ by using constant and dynamic permeability. For $x=1.5 \mathrm{~L}$, the vertical distribution of $p-P_{b}$ is given in Fig. 21 . The results using constant permeability agrees exactly with the analytical solution by Yamamoto et al. (1978), which again validates the numerical code developed in this paper. The dynamic permeability is observed to reduce the value of $p-P_{b}$ under the wave trough, thus ease the unreasonable behavior that the wave-induced pore pressure exceeds the initial vertical effective stress.

For $\sigma_{v 0}^{\prime}-\left(p-P_{b}\right)$, the contours are given by Fig. 22 and the vertical distribution along $x=1.5 \mathrm{~L}$ is given in Fig. 23. The minimum values of $\sigma_{v 0}^{\prime}-\left(p-P_{b}\right)$ is $-9.04 \mathrm{kPa}$ by using constant permeability. The negative value is rather large. If dynamic permeability is used, the minimum value is reduced to $-2.00 \mathrm{kPa}$. The improvement is apparent. By finding the isoline of $\sigma_{v 0}^{\prime}-\left(p-P_{b}\right)=0$, the liquefaction zone can be determined, as shown in Fig. 22. Fig. 24 further compares the liquefaction zones. The traditional model using constant permeability is observed to overestimate the liquefaction potential.

Fig. 25 presents the contours of the excess pore pressure ratio $r_{u} \cdot r_{u}$ decreases with the increase of soil depth. The maximum values appear near the seabed surface, with specific values of $r_{\mathrm{u}}=5.78$ and 1.72 for constant and dynamic permeability, respectively. To correct the behavior of $r_{u}>1.0$, permeability increase in the liquefaction zone is needed. Fig. 26 shows the distribution of permeability ratio in the

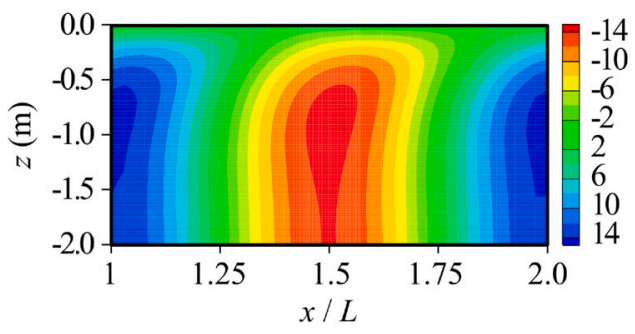

(a)

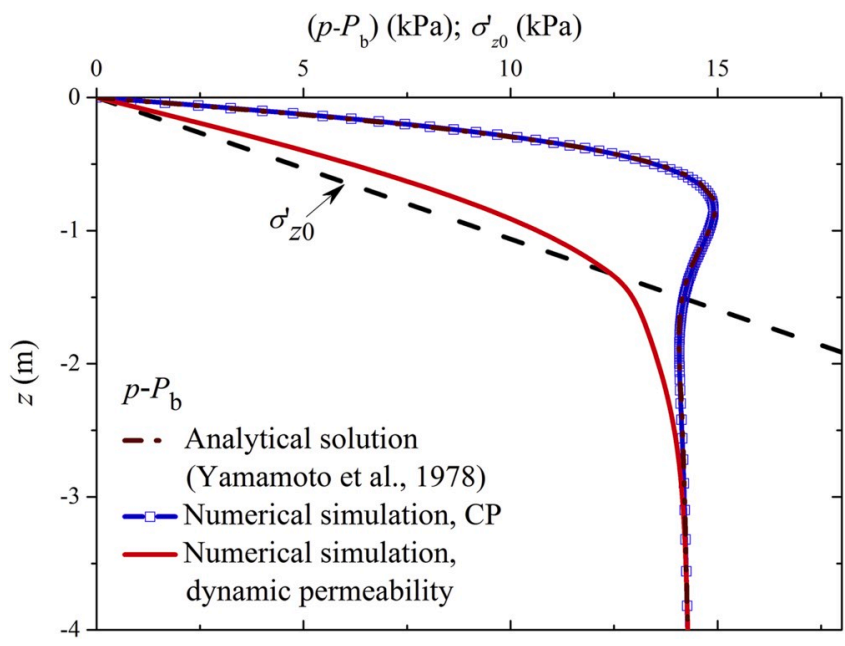

Fig. 21. Vertical distribution of $p-P_{b}$ in the 2-D simulation.

seabed when the dynamic permeability is utilized. The permeability distribution pattern is similar to that of $r_{u}$. The maximum value of dynamic permeability is 7.51 times the initial value.

\subsubsection{Nonlinear convergence performance}

Fig. 27(a) shows the iteration numbers cost by each time step. The Newton-Raphson method requires averagely more than 10 iterations to achieve the convergence. For two typical time steps marked in Fig. 27 (a), Fig. 27(b) shows the convergence process. Compared with the theoretically quadratic convergence of the Newton-Raphson method, the performance in Fig. 27(b) is rather poor.

As supplementary results, Fig. 28 gives the convergence performance by using DP2 and DP3. Numerical divergences appear at the first time

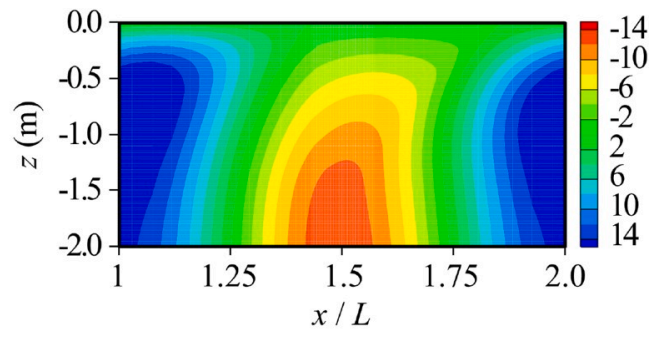

(b)

Fig. 20. Contours of $p-P_{b}$ by (a) CP; and (b) dynamic permeability. 


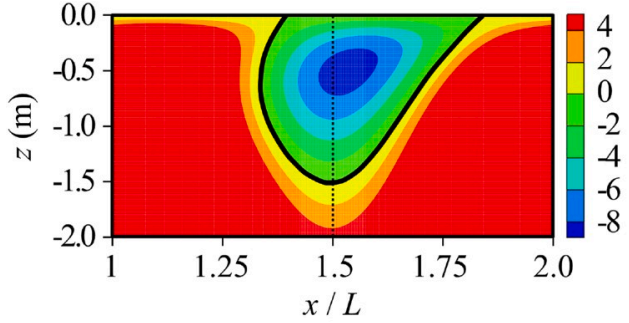

(a)

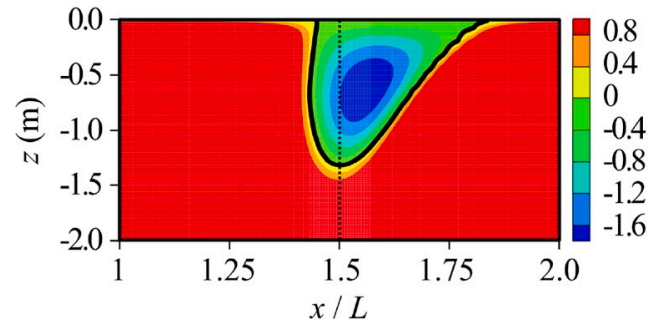

(b)

Fig. 22. Contours of $\sigma_{v 0}^{\prime}-\left(p-P_{b}\right)$ by (a) CP; and (b) dynamic permeability.

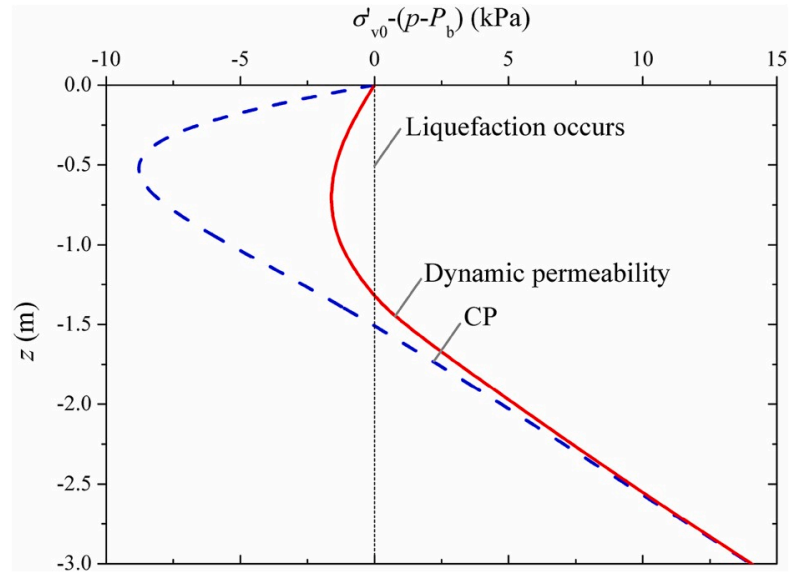

Fig. 23. Vertical distribution of $\sigma_{v 0}^{\prime}-\left(p-P_{b}\right)$ in the 2-D simulation.

step. Neither convergence nor meaningful results can be obtained, because these two parameter groups introduce an extremely strong nonlinearity for 2-D simulations. Novel numerical treatments should be developed to overcome this problem.

\section{Conclusions}

The wave-induced pore pressure and associated seabed liquefaction are crucial to analyzing the stability of offshore foundations in coastal locations. In this study, a dynamic permeability model is proposed to numerically analyze the wave-induced seabed response. The following conclusions can be drawn.

(1) The increase in the permeability coefficient of saturated sand during liquefaction can be up to 5-7 times the initial value, due to an easier and shorter path for the pore water to readily flow through the soil during liquefaction. On the basis of existing

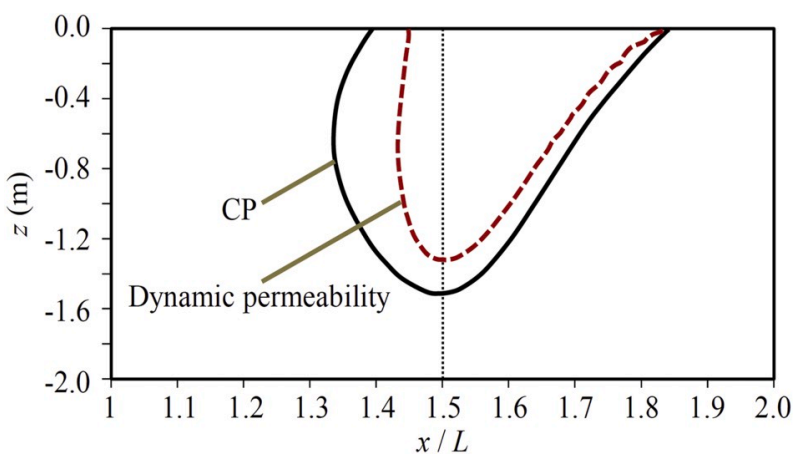

Fig. 24. The wave-induced liquefaction zone.

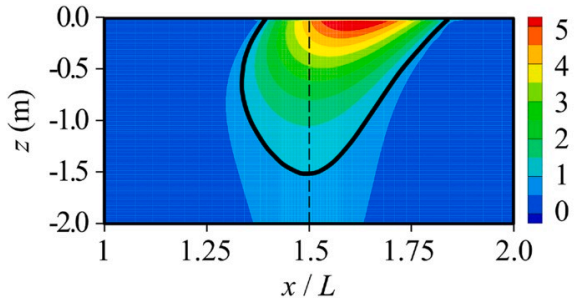

(a)

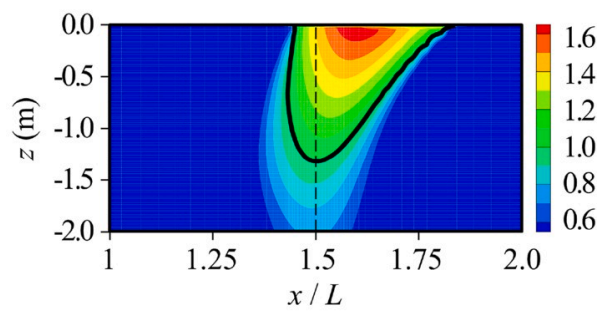

(b)

Fig. 25. Contours of $r_{u}$ by (a) constant; and (b) dynamic permeability.

dynamic permeability models ever used for seismic liquefaction, we add a starting value of the excess pore pressure ratio to control when the soil permeability begins to increase. This starting value proves to be crucial for the dynamic permeability model to properly reproduce the wave-seabed interaction.

(2) The nonlinearity introduced by the dynamic permeability is solved in an in-house finite element code by an iterative procedure based on the Newton-Raphson method. The in-house code is compared with the constant-permeability analytical solutions and then applied to simulate the 1-D cylinder tests by using both constant and dynamic permeability. When there is no liquefaction, the dynamic permeability model obtains identical results to

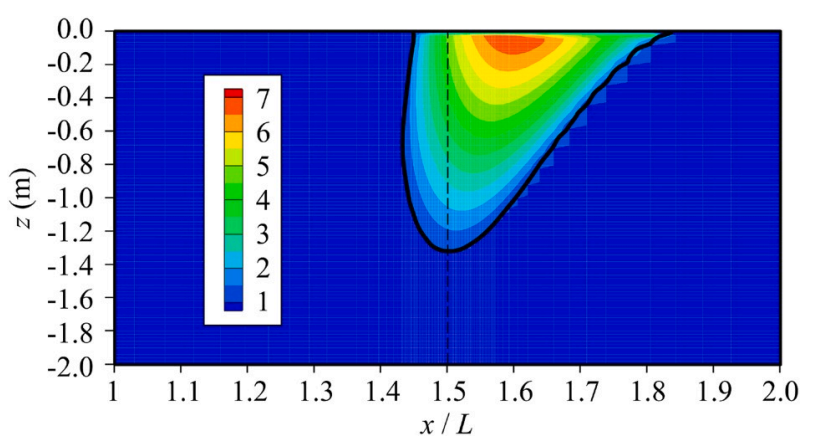

Fig. 26. Contour of the permeability ratio $k / k_{0}$ by using dynamic permeability. 


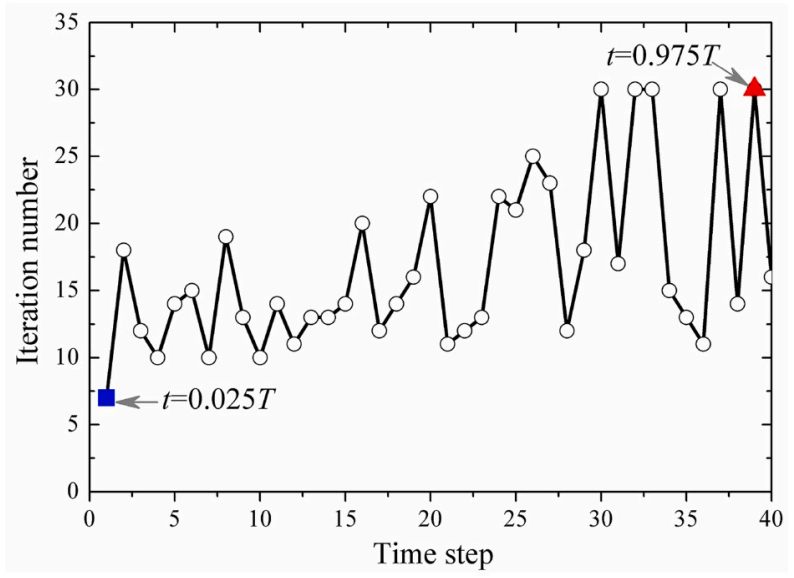

(a)

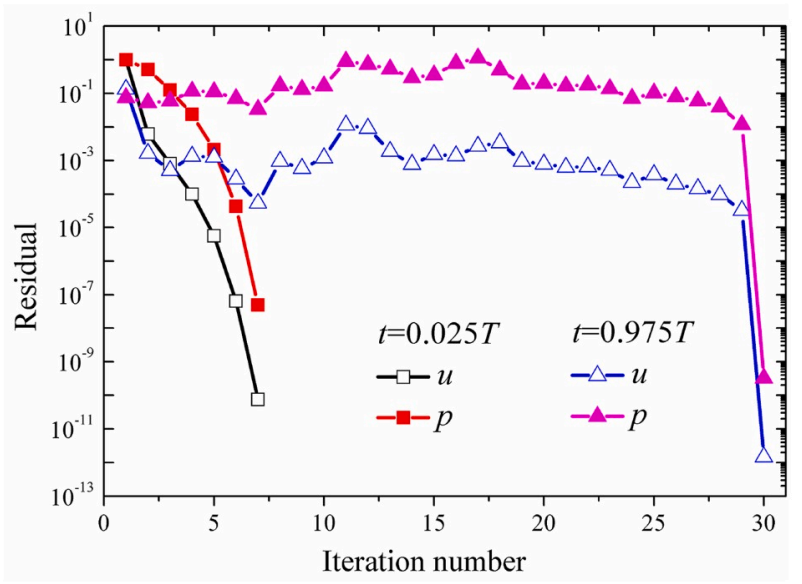

(b)

Fig. 27. Convergence performance by using $c_{1}=10, c_{2}=1$ and $r_{u}^{\mathrm{cr}}=1$ : (a) iteration number versus time step; (b) residual versus iteration.

the conventional constant permeability model. But for the case where liquefaction is observed, the dynamic permeability has significant influence. In the liquefied zone, the unreasonable tensile stress obtained by the constant permeability is remarkably alleviated by using dynamic permeability. The conventional model with constant permeability overestimates the liquefaction potential, due to its unreasonable permitting of tensile stress in sandy seabed. For all cases presented in this paper, the maximum liquefaction depth given by constant permeability can be up to two times the value by dynamic permeability.

(3) The effects of model parameters with respect to dynamic permeability are investigated. If the permeability can increase large enough when soil liquefies, the final numerical results are no longer sensitive to the specific values of the model parameters introduced by the dynamic permeability. This performance is similar to the penalty method within the constrained variational principle. However, further investigations of the nonlinear convergence behavior show that larger permeability increase can lead to lower convergence rates or even numerical divergences. Advanced numerical strategies are now in progress to improve the performance.

The new concept of removing unreasonable tensile stress in a sandy seabed provides a wide range of possibilities to improve existing models which treated the seabed as an invariant medium. This concept is

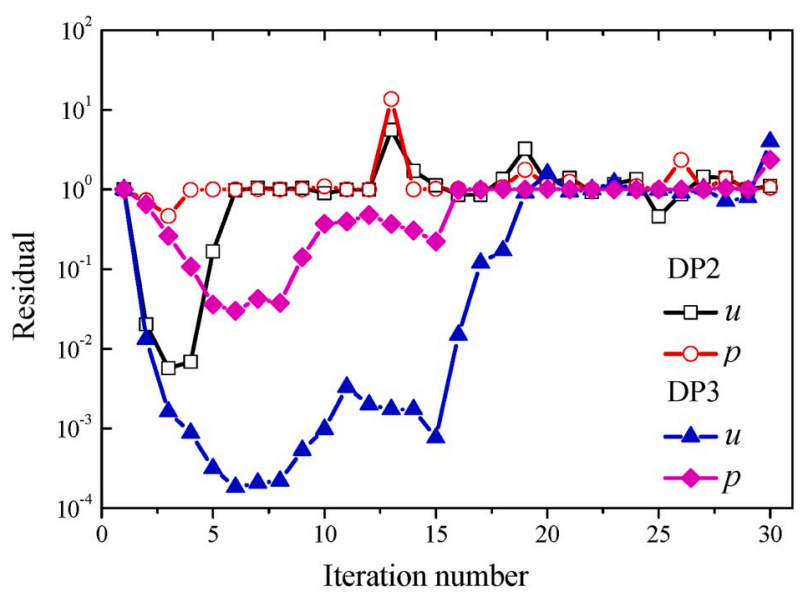

(a)

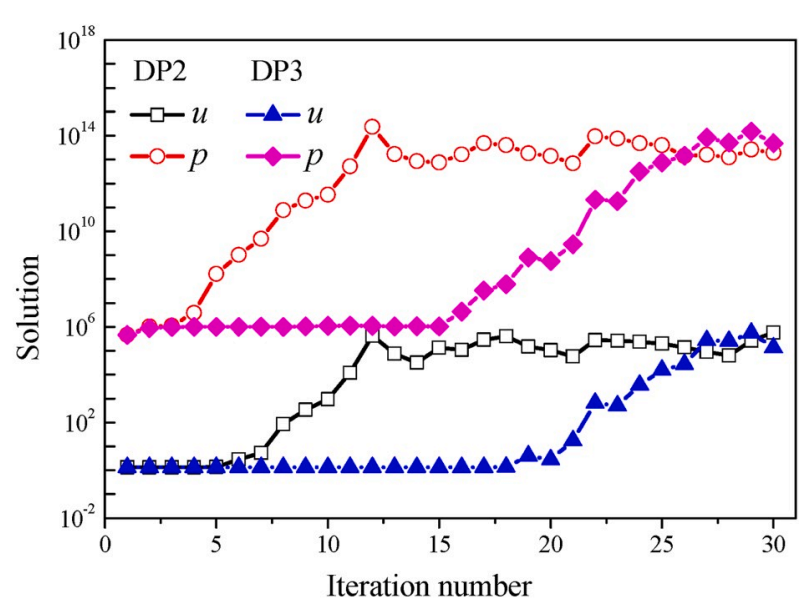

(b)

Fig. 28. Convergence performance by using DP2 and DP3: (a) residual versus iteration; (b) solution versus iteration.

numerically implemented in this paper as the permeability variation. Other attempts are also worthy of further investigations, such as decreasing the solid-phase resistance during liquefaction. Moreover, the continuous porous-medium assumption applied in this study for liquefied soil also calls for further investigations.

\section{Declaration of competing interest}

The authors declare that they have no known competing financial interests or personal relationships that could have appeared to influence the work reported in this paper.

\section{CRediT authorship contribution statement}

Mo-Zhen Zhou: Methodology, Validation, Writing - original draft. Dong-Sheng Jeng: Formal analysis, Writing - review \& editing. WenGang Qi: Conceptualization, Visualization.

\section{Acknowledgements}

The authors would like to thank the National Natural Science Foundation of China (Grant Nos. 51808034 and 11972036) for financial support. 


\section{References}

Adamidis, O., Madabhushi, G.S.P., 2016. Post-liquefaction reconsolidation of sand. Proc. R. Soc. A 472, 20150745.

Arulanandan, K., Sybico Jr., J., 1992. Post-liquefaction Settlement of Sand. The Wroth Memorial Symposium. Oxford University, England, pp. 94-110.

Biot, M.A., 1941. General theory of three-dimensional consolidation. J. Appl. Phys. 26 (2), 155-164.

Carman, P.C., 1956. Flow of Gases through Porous Media. Butterworths, London.

Celli, D., Li, Y.Z., Ong, M.C., Di Risio, M., 2019. The role of submerged berms on the momentary liquefaction around conventional rubble mound breakwaters. Appl. Ocean Res. 85, 1-11.

Chapuis, R.P., Aubertin, M., 2003. On the use of the Kozeny-Carman equation to predict the hydraulic conductivity of soils. Can. Geotech. J. 40 (3), 616-628.

Chen, W.Y., Liu, C.L., Duan, L.L., Qiu, H.M., Wang, Z.H., 2019. 2D numerical study of the stability of trench under wave action in the immersing process of tunnel element. J. Mar. Sci. Eng. 7 (3), 57.

Gardner, W., 1956. Mathematics of isothermal water conduction in unsaturated soils. Highway. Res. Board. Spec. Report 40, 78-87.

Ghassemi, A., Pak, A., 2011. Pore scale study of permeability and tortuosity for flow through particulate media using Lattice Boltzmann method. Int. J. Numer. Anal. Methods GeoMech. 35 (8), 886-901.

Ha, I.S., Park, Y.H., Kim, M.M., 2003. Dissipation pattern of excess pore pressure after liquefaction in saturated sand deposits. Transport. Res. Rec. 1821 (1), 59-67.

Haigh, S.K., Eadington, J., Madabhushi, S.P.G., 2012. Permeability and stiffness of sands at very low effective stresses. Geotechnique 62 (1), 69-75.

Han, S., Jeng, D.-S., Tsai, C.C., 2019. Response of a porous seabed around an immersed tunnel under wave loading: meshfree model. J. Mar. Sci. Eng. 7 (10), 369.

Hsu, J.R.C., Jeng, D.-S., 1994. Wave-induced soil response in an unsaturated anisotropic seabed of finite thickness. Int. J. Numer. Anal. Methods GeoMech. 18 (11), 785-807.

Jeng, D.-S., Lin, Y.S., 1996. Finite element modelling for water waves-soil interaction. Soil Dynam. Earthq. Eng. 15 (5), 283-300, 1996.

Jeng, D.-S., Seymour, B.R., 1997. Response in seabed of finite depth with variable permeability. J. Geotech. Geoenviron. Eng. 123 (10), 902-911.

Jeng, D.-S., Lin, Y.S., 1999. Wave-induced pore pressure around a buried pipeline in Gibson soil: finite element analysis. Int. J. Numer. Anal. Methods GeoMech. 23 (13), 1559-1578.

Jeng, D.-S., Cha, D.H., Lin, Y.S., Hu, P.S., 2001. Wave-induced pore pressure around a composite breakwater. Ocean Eng. 28 (10), 1413-1435.

Jeng, D.-S., 2018. Mechanics of Wave-Seabed-Structure Interactions: Modelling, Processes and Applications. Cambridge University Press, Cambridge.

Kozeny, J., 1927. Ueber kapillare leitung des wassers im boden. Sitzungsber Akad. Wiss, Wien 136, 271-306.

Li, K., Guo, Z., Wang, L.Z., Jiang, H.Y., 2019. Effect of seepage flow on shields number around a fixed and sagging pipeline. Ocean Eng. 172, 487-500.

Li, W.T., Wei, C.F., 2018. Stabilized low-order finite elements for strongly coupled poromechanical problems. Int. J. Numer. Methods Eng. 115 (5), 531-548.

Li, Y., Ong, M.C., Tang, T., 2018. Numerical analysis of wave-induced poro-elastic seabed response around a hexagonal gravity-based offshore foundation. Coast. Eng. 136, 81-95.

Li, Y., Ong, M.C., Tang, T., 2020. A numerical toolbox for wave-induced seabed response

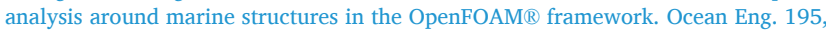
106678.

Lin, Z.B., Pokrajac, D., Guo, Y.K., Jeng, D.-S., Tang, T., Rey, N., Zheng, J.H., Zhang, J.S., 2017. Investigation of nonlinear wave-induced seabed response around mono-pile foundation. Coast. Eng. 121, 197-211.

Liu, B., Jeng, D.-S., Ye, G.L., Yang, B., 2015. Laboratory study for pore pressures in sandy deposit under wave loading. Ocean Eng. 106, 207-219.

Liu, Y.F., Jeng, D.-S., 2019. Pore scale study of the influence of particle geometry on soil permeability. Adv. Water Resour. 129, 232-249.

List, F., Radu, F.A., 2016. A study on iterative methods for solving Richards' equation. Comput. Geosci. 20 (2), 341-353.
Madsen, O.S., 1978. Wave-induced pore-pressures and effective stresses in a porous bed. Geotechnique 28 (4), 377-393.

Mei, C.C., Foda, M.A., 1981. Wave-induced response in a fluid-filled poro-elastic solid with a free surface-a boundary layer theory. Geophys. J. R. Astron. Soc. 66, 597-631.

Qi, W.G., Gao, F.P., 2018. Wave induced instantaneously-liquefied soil depth in a noncohesive seabed. Ocean Eng. 153, 412-423.

Qi, W.G., Li, C.F., Jeng, D.-S., Gao, F.P., Liang, Z., 2019. Combined wave-current induced excess pore-pressure in a sandy seabed: flume observations and comparisons with theoretical models. Coast. Eng. 147, 89-98.

Qi, W.G., Shi, Y.M., Gao, F.P., 2020. Uplift soil resistance to a shallowly-buried pipeline in the sandy seabed under waves: poro-elastoplastic modeling. Appl. Ocean Res. 95, 102024.

Sakai, T., Hatanaka, K., Mase, H., 1992. Wave-induced effective stress in seabed and its instantaneous liquefaction. J. Waterw. Port, Coast. Ocean Eng. 118 (2), 202-206.

Sassa, S., Sekiguchi, H., 2001. Analysis of wave-induced liquefaction of sand beds. Geotechnique 51 (2), 115-126.

Shahir, H., Mohammadi-Haji, B., Ghassemi, A., 2014. Employing a variable permeability model in numerical simulation of saturated sand behavior under earthquake loading. Comput. Geotech. 55, 211-223.

Shahir, H., Pak, A., Taiebat, M., Jeremić, B., 2012. Evaluation of variation of permeability in liquefiable soil under earthquake loading. Comput. Geotech. 40, 74-88.

Sui, T.T., Zhang, C., Guo, Y.K., Zheng, J.H., Jeng, D.S., Zhang, J.S., Zhang, W., 2016. Three-dimensional numerical model for wave-induced seabed response around mono-pile. Ships Offshore Struct. 11, 667-678.

Sui, T., Zheng, J., Zhang, C., Jeng, D.-S., Zhang, J., Guo, Y., He, R., 2017. Consolidation of unsaturated seabed around an inserted pile foundation and its effects on the waveinduced momentary liquefaction. Ocean Eng. 131, 308-321.

Sui, T., Jin, Y., Wang, Z., Zhang, C., Shi, J., 2019. Effects of the soil property distribution gradient on the wave-induced response of a non-homogeneous seabed. J. Mar. Sci. Eng. 7, 281.

Sumer, B.M., Hatipoglu, F., Fredsoe, J., Sumer, S.K., 2006. The sequence of sediment behaviour during wave-induced liquefaction. Sedimentology 53 (3), 611-629.

Sumer, B.M., 2014. Liquefaction Around Marine Structures. World Scientific, Singapore.

Ueng, T.S., Wang, Z.F., Chu, M.C., Ge, L., 2017. Laboratory tests for permeability of sand during liquefaction. Soil Dynam. Earthq. Eng. 100, 249-256.

Wang, W., Zhou, M.Z., Zhang, B.Y., Peng, C., 2019. A dual mortar contact method for porous media and its application to clay-core rockfill dams. Int. J. Numer. Anal. Methods GeoMech. 43 (9), 1744-1769.

Wang, B., Zen, K., Chen, G.Q., Zhang, Y.B., Kasama, K., 2013. Excess pore pressure dissipation and solidification after liquefaction of saturated sand deposits. Soil Dynam. Earthq. Eng. 49, 157-164.

Wu, S., Jeng, D.-S., 2019. Effects of dynamic soil permeability on the wave-induced seabed response around a buried pipeline. Ocean Eng. 186, 106132.

Wu, S., Jeng, D.-S., Seymour, B.R., 2020. Numerical modelling of consolidation-induced solute transport in unsaturated soil with dynamic hydraulic conductivity and degree of saturation. Adv. Water Resour. 135, 103466.

Yamamoto, T., Koning, H., Sellmeijer, H., Hijum, E.V., 1978. On the response of a poroelastic bed to water waves. J. Fluid Mech. 87 (1), 193-206.

Ye, J.H., Jeng, D.S., 2012. Response of porous seabed to nature loadings: waves and currents. J. Eng. Mech. 138 (6), 601-613.

Zen, K., Yamazaki, H., 1990. Mechanism of wave-induced liquefaction and densification in seabed. Soils Found. 30 (4), 90-104.

Zhang, C., Sui, T., Zheng, J., Xie, M., Nguyen, V.T., 2016. Modelling wave-induced 3D non-homogeneous seabed response. Appl. Ocean Res. 61, 101-114.

Zhang, J., Tong, L., Zheng, J., He, R., Guo, Y., 2018. Effects of soil resistance damping on wave-induced pore pressure accumulation around a composite breakwater. J. Coast Res. 34 (3), 573-585.

Zhou, M.Z., Zhang, B.Y., Peng, C., 2018. Numerical evaluation of soft inter-slab joint in concrete-faced rockfill dam with dual mortar finite element method. Int. J. Numer. Anal. Methods GeoMech. 42 (5), 781-805. 